\title{
Spontaneous Superrotation and the Role of Kelvin Waves in an Idealized Dry GCM
}

\author{
SAMuel F. PotTer AND GeOFFrey K. VAllis* \\ Atmospheric and Oceanic Sciences Program, Princeton University, Princeton, New Jersey \\ JONATHAN L. MitCHELL \\ Earth and Space Sciences, Atmospheric and Oceanic Sciences, University of California, Los Angeles, Los Angeles, California
}

(Manuscript received 20 May 2013, in final form 20 September 2013)

\begin{abstract}
The nondimensional parameter space of an idealized dry primitive equation model is explored to find superrotating climate states. The model has no convective parameterization and is forced using a simple thermal relaxation to a prescribed radiative equilibrium temperature. It is demonstrated that, of four nondimensional parameters that determine the model's state, only the thermal Rossby number has a significant effect on superrotation. The mode that drives the transition to superrotation in an intermediate-thermalRossby-number atmosphere is shown to behave like a Kelvin wave in the tropics.
\end{abstract}

\section{Introduction}

An atmosphere is superrotating if at some location the winds have greater angular momentum than that of the planet at the equator $\Omega a^{2}$, where $\Omega$ is the rotation rate and $a$ is the planetary radius. This condition becomes more difficult to satisfy farther poleward as the lower bound for superrotating winds, $u_{s}=\Omega a \sin ^{2} \phi / \cos \phi$, where $\phi$ is latitude, increases rapidly with latitude. Furthermore, if an atmosphere is superrotating in midlatitudes, but not at the equator, then it will normally be inertially or centrifugally unstable. Thus, superrotation usually implies equatorial superrotation. Although Earth's troposphere is not, on average, superrotating, at times it may be, and there has long been interest in understanding superrotation in Earthlike atmospheric models (e.g., Suarez and Duffy 1992; Saravanan 1993). Titan, the sixth moon of Saturn, has a terrestrial atmosphere (i.e., an atmosphere with a distinct, often rocky lower boundary) that superrotates, as does Venus and the gas giants

\footnotetext{
* Current affiliation: Department of Mathematics, University of Exeter, Exeter, United Kingdom.

Corresponding author address: Sam Potter, Atmospheric and Oceanic Sciences Program, Princeton University, 300 Forrestal Road, Princeton, NJ 08540-6654.

E-mail: spotter@princeton.edu
}

Jupiter and Saturn. Superrotation has also been seen in exoplanet modeling studies for both terrestrial exoplanets (e.g., Joshi et al. 1997; Merlis and Schneider 2010) and gas giant exoplanets (e.g., Showman et al. 2009; Heng et al. 2011; Rauscher and Menou 2012).

By Hide's theorem (Hide 1969) - essentially an application of the general result that there can be no internal extremum of a Lagrangian-conserved quantity if that quantity is diffused (Vallis 2006) - superrotation in the time mean must be maintained by eddy momentum fluxes. Explicit wave forcing at the equator may give rise to superrotation-as in the primitive equation experiments of Suarez and Duffy (1992), Saravanan (1993), and Kraucunas and Hartmann (2005) - in which the waves were generated by a zonally asymmetric tropical heating. Arnold et al. (2012) also found superrotation in a dynamical core forced with a moving tropical diabatic heating and identified an equatorial Rossby wave resonance as being responsible for eddy momentum flux convergence at the equator. Wave forcing also gave rise to superrotation in the 1.5-layer shallow-water model of Showman and Polvani (2010).

In the studies cited above, the cause of the superrotationwave forcing at the equator-is made explicit. There have been fewer studies of the mechanism of spontaneous superrotation in Earthlike models, in which equatorial westerlies exist in the time mean in the absence of zonally asymmetric forcing. If there were a source of Rossby waves at the equator, then a superrotating state 
might be expected to emerge, since if the Rossby waves were able to propagate away momentum would converge on the source of the Rossby waves and, if strong enough, generate an eastward flow. In midlatitudes, baroclinic instability is such a source and at the equator convective activity is a potential source, but the circumstances under which convection can generate sufficiently strong Rossby waves, and whether such Rossby waves can propagate sufficiently far from the equator before breaking, are not well known. Saravanan (1993) did find that superrotation persists even after the tropical forcing was turned off as a consequence of a westerly torque produced by eddying activity at the equator, although the nature and robustness of the eddies giving rise to the superrotation was not explicitly identifiedindeed, Kraucunas and Hartmann (2005) suggested that the mechanism suggested by Saravanan does not apply in multilevel models.

More recent works suggest that thinking of an equatorial westerly jet in the same way as a midlatitude Rossby wave-driven jet may be incorrect. Showman and Polvani (2011) showed that momentum fluxes arising from a stationary equatorial forcing are the result of equatorial shallow water waves, not meridionally propagating Rossby waves. Specifically, the equatorial Rossby wave-Kelvin wave structure of the Gill response to a heating generates equatorward momentum fluxes that are likely the cause of superrotation seen in models with stationary wave-like equatorial heating (e.g., Suarez and Duffy 1992; Saravanan 1993; Kraucunas and Hartmann 2005). Other studies have found that equatorial Rossby waves (e.g., Liu and Schneider 2011; Arnold et al. 2012) or Kelvin waves (e.g., Iga and Matsuda 2005) play a crucial role in the generation of superrotation. In this study, we aim partly to demonstrate the need for thinking of equatorial westerly jet dynamics as distinct from the dynamics of eddy-driven midlatitude jets.

Williams (2003) induced spontaneous superrotation in a dynamical core by changing both the functional form of the radiative cooling profile and the planetary rotation rate. $\mathrm{He}$ increased the exponent of the latitudinal dependence of the thermal forcing from $\cos ^{2}$ to $\cos ^{n}$, where $n=4,8,16,32$, and 64 , and found that superrotation was the preferred state in the lower-rotation, higher- $n$-parameter regime. It was hypothesized that the superrotation was the result of barotropic instability on the equatorward side of the subtropical jets.

Mitchell and Vallis (2010, hereafter MV10) found that superrotation robustly exists in models of terrestrial atmospheres with large thermal Rossby number. In their study, the thermal Rossby number was varied via the planetary radius and a planet with a smaller radius was found to be more likely to superrotate. They found that the circulation of the large-thermal-Rossby-number atmosphere was dominated by a global wavenumber- 1 mode that drove the superrotation, but they were unable to reach a definitive conclusion as to what drove the superrotation. Although barotropic instability seemed to play a role, it is unlikely that this is the entire story since barotropic Rossby waves converge momentum into their source region and draw momentum out of the region where they break.

While studying hothouse climates with a full-physics AGCM coupled to a slab ocean, Caballero and Huber (2010) found that as $\mathrm{CO}_{2}$ was increased in their model the eddy flux of momentum into the tropics grew until the atmosphere began to superrotate. The presence of a hydrological cycle and convection scheme was key in their results, and the mode driving superrotation strongly resembled the Madden-Julian oscillation (MJO). The importance of the MJO to the momentum balance of the tropics was also shown by Lee (1999), who demonstrated that all that was required to make an AGCM superrotate was to turn off the seasonal cycle. In permanent equinox, the model weakly superrotated and eddy fluxes into the tropics were dominated by the MJO.

With all of the above studies in mind, our paper has two goals. The first is to extend the results of MV10 to explore further the nondimensional parameter space of a dry dynamical core with the goal of finding what parameters are most important for the momentum balance of the equatorial troposphere. The second is an attempt to identify the modes of variability that drive superrotation in a dry model. We begin with a brief overview of the model in section 2, describe the simulations in sections 3 and 4, analyze the results in section 5, briefly discuss similar spectral results in other contexts in section 6 , and conclude in section 7 .

\section{Methods}

\section{a. Model description}

We use the spectral dynamical core from the Geophysical Fluid Dynamics Laboratory's Flexible Modeling System (FMS). The FMS dry dynamical core is forced with "Held-Suarez" idealized physics (Held and Suarez 1994). All of the runs use T42 spectral resolution with 20 equally spaced sigma levels and a fourth-order hyperdiffusion. We begin by nondimensionalizing the primitive equations to find the dynamical core's controlling parameters. The primitive equation nondimensional parameters are found in the zonal momentum and thermodynamic equations:

$$
\begin{aligned}
\frac{\partial u}{\partial t}+(\mathbf{v} \cdot \nabla) u+\omega \frac{\partial u}{\partial p}= & f v+\frac{u v \tan \phi}{a} \\
& -\frac{1}{a \cos \phi} \frac{\partial \Phi}{\partial \lambda}-r u \text { and }
\end{aligned}
$$


TABLE 1. Key dimensional and nondimensional parameters of the standard Held-Suarez forced dynamical core.

\begin{tabular}{lccccc}
\hline \hline \multicolumn{1}{c}{$a$} & $\Omega$ & $R$ & $\Delta_{h}$ & $\Delta_{v}$ \\
\hline $6.371 \times 10^{6} \mathrm{~m}$ & $7.292 \times 10^{-5} \mathrm{~s}^{-1}$ & $287 \mathrm{~J} \mathrm{~K}^{-1} \mathrm{~kg}^{-1}$ & $60 \mathrm{~K}$ & $10 \mathrm{~K}$ & $1 \mathrm{day}^{-1}$ \\
$\tau_{r}$ & $R_{o}$ & $E$ & $\tau$ & $\mu$ \\
\hline 40 day $^{-1}$ & 0.02 & 0.079 & 504 & 0.17 \\
\hline
\end{tabular}

$$
\frac{\partial \theta}{\partial t}+(\mathbf{v} \cdot \nabla) \theta+\omega \frac{\partial \theta}{\partial p}=-\frac{\theta-\theta^{*}}{\tau_{r}} .
$$

In the above equations, $(\mathbf{v} \cdot \nabla)=(u / a \cos \phi) \partial / \partial \lambda+(v / a) \partial / \partial \phi$ is the horizontal advective component of the total derivative where $p$ is pressure and $\lambda$ is longitude. The final term in the zonal momentum equation is Rayleigh drag, with time scale $1 / r$. Likewise, the final term in the thermodynamic equation is a Newtonian cooling with the permanent-equinox Held-Suarez relaxation profile $\theta^{*}$, in potential temperature $\theta$, with cooling time scale $\tau_{r}$ :

$$
\begin{aligned}
\theta^{*}(\phi, p)= & \max \left\{200\left(p_{o} / p\right)^{\kappa},\left[\theta_{0}-\Delta_{h} \sin ^{2} \phi\right.\right. \\
& \left.\left.-\Delta_{v} \log \left(\frac{p}{p_{0}}\right) \cos ^{2} \phi\right]\right\},
\end{aligned}
$$

where $p_{0}$ is the surface pressure, $\kappa=g / c_{p}, \Delta_{h}$ is the equator-to-pole temperature forcing gradient, and $\Delta_{v}$ is the surface-to-top-of-atmosphere temperature forcing gradient. The hydrostatic and continuity equations and the ideal gas equation of state complete the set.

The equations are nondimensionalized by scaling length with $a$, time by $1 /(2 \Omega)$, and geopotential height by $\Omega a U$. All potential temperatures except the one appearing in the vertical advection term are scaled by $\Delta_{h}$. The potential temperature that appears in the $\omega \partial \theta / \partial p$ term in Eq. (2) is scaled with $\Delta_{v}$ to reflect the difference in vertical and horizontal temperature scales. The horizontal wind scale $U \sim R \Delta_{h} /(2 \Omega a)$ is derived from thermal wind balance, where $R$ is the dry specific gas constant and the pressure velocity $\omega$ is scaled using mass continuity, giving $\omega \sim U \delta p / a$. While thermal wind is not a good approximation for large Rossby numbers, it is still useful as a control parameter. The term $\delta p$ is a scale height in pressure coordinates.

With these scales, Eqs. (1) and (2) become

$$
\begin{aligned}
\frac{\partial u}{\partial t} & +R_{o}\left[(\mathbf{v} \cdot \nabla) u+\omega \frac{\partial u}{\partial p}\right] \\
& =\hat{f} v+R_{o} u v \tan \phi-\frac{1}{2 \cos \phi} \frac{\partial \Phi}{\partial \lambda}-E u \text { and }
\end{aligned}
$$

$$
\frac{\partial \theta}{\partial t}+R_{o}\left[(\mathbf{v} \cdot \nabla) \theta+\mu \omega \frac{\partial \theta}{\partial p}\right]=-\frac{\theta-\theta^{*}}{\tau} .
$$

All terms are now nondimensional, with $\hat{f}=\sin \phi$ being a nondimensional Coriolis parameter; $R_{o}=\left(R \Delta_{h}\right) /$ $(2 \Omega a)^{2}$ is the thermal Rossby number, $E=r /(2 \Omega)$ is the Ekman number, $\tau=2 \Omega \tau_{r}$ is a nondimensional radiative time scale, and $\mu=\Delta_{v} / \Delta_{h}$ is the ratio of vertical to horizontal temperature forcing gradients in the HeldSuarez forcing profile. Note that the $200-K$ definition of the Held-Suarez stratosphere means that changing $\Delta_{v}$ will change both the stratification and the tropopause height. The value of the relevant constants and nondimensional parameters for the Earth-control Held-Suarez-forced dynamical core can be found in Table 1.

In this paper, we discuss model integrations where each nondimensional parameter has been varied separately (e.g., in the thermal Rossby number runs, $R_{o}$ is changed while $E, \tau$, and $\mu$ are held fixed). As the code itself has dimensional parameters, the Rossby number is changed through the planetary radius, the Ekman number is changed through the Rayleigh drag time scale, the nondimensional radiative time scale is changed via the Newtonian cooling time scale, and the ratio of vertical to horizontal temperature gradients is changed through the vertical temperature forcing gradient. A list of experiments is shown in Table 2.

\section{b. Spectral analyses}

We use two different spectral methods to analyze tropical variability in our model results. First, we decompose the tropical geopotential height field in spectral space following closely Wheeler and Kiladis (1999). 128-day instantaneous slices of geopotential height equatorward of $15^{\circ}$ along a single-latitude band and a specific pressure level are Fourier transformed in space and time. The absolute magnitudes of the spectral components are calculated for resolved zonal wavenumbers and frequencies. These spectral data are smoothed 10 times with a 1-2-1 filter and the raw spectral data are divided by the smoothed data to highlight spectral peaks that denote wavelike activity. 
TABLE 2. List of parameter values for all experiments. Each paired set of rows first displays the nondimensional parameter that was changed with the corresponding dimensional parameter value below.

\begin{tabular}{|c|c|c|c|c|c|c|c|c|c|c|c|}
\hline \multirow{2}{*}{$\begin{array}{c}\text { Parameter } \\
R_{o} \\
a\end{array}$} & \multicolumn{4}{|l|}{ Units } & \multicolumn{7}{|c|}{ Control } \\
\hline & $\mathrm{m}$ & & & & & & $\begin{array}{c}4.8 \times 10^{-3} \\
1.3 \times 10^{7}\end{array}$ & $\begin{array}{c}0.020 \\
6.371 \times 10^{6}\end{array}$ & $\begin{array}{c}0.103 \\
2.8 \times 10^{6}\end{array}$ & $\begin{array}{c}1.00 \\
9.0 \times 10^{5}\end{array}$ & $\begin{array}{c}10.3 \\
2.8 \times 10^{5}\end{array}$ \\
\hline $\begin{array}{l}E \\
r\end{array}$ & day $^{-1}$ & & & & $\begin{array}{c}4.0 \times 10^{-4} \\
0.005\end{array}$ & $\begin{array}{c}1.6 \times 10^{-3} \\
0.02\end{array}$ & $\begin{array}{c}7.9 \times 10^{-3} \\
0.1\end{array}$ & $\begin{array}{c}0.079 \\
1\end{array}$ & $\begin{array}{c}0.79 \\
10\end{array}$ & $\begin{array}{l}1.6 \\
20\end{array}$ & $\begin{array}{c}7.9 \\
100\end{array}$ \\
\hline $\begin{array}{l}\tau \\
\tau_{r}\end{array}$ & day & $\begin{array}{l}0.063 \\
0.005\end{array}$ & $\begin{array}{l}0.126 \\
0.01\end{array}$ & $\begin{array}{l}0.63 \\
0.05\end{array}$ & $\begin{array}{l}1.26 \\
0.1\end{array}$ & $\begin{array}{c}12.6 \\
1\end{array}$ & $\begin{array}{c}101 \\
8\end{array}$ & $\begin{array}{c}504 \\
40\end{array}$ & $\begin{array}{c}2520 \\
200\end{array}$ & $\begin{array}{c}12600 \\
1000\end{array}$ & \\
\hline $\begin{array}{l}\mu \\
\Delta_{v}\end{array}$ & $\mathrm{~K}$ & & & & & $\begin{array}{c}0.0017 \\
0.1\end{array}$ & $\begin{array}{c}0.017 \\
1\end{array}$ & $\begin{array}{c}0.17 \\
10\end{array}$ & $\begin{array}{c}0.83 \\
50\end{array}$ & $\begin{array}{l}1.7 \\
100\end{array}$ & \\
\hline
\end{tabular}

Second, we follow Hayashi (1982) and Randel and Held (1991) to compute $\mathcal{S}=-1 /\left(a \cos ^{2} \phi\right) \partial / \partial \phi\left(\left[\overline{u^{\prime} v^{\prime}}\right] \cos ^{2} \phi\right)$, the acceleration of the mean zonal winds by meridional eddy momentum divergence of transient eddies, as a function of frequency and zonal wavenumber. Primes denote a deviation from the time mean, the overbar denotes a time mean, and square brackets denote a zonal mean. Since our forcing is zonally symmetric and fixed in time we do not need to consider zonal deviations in our calculations. A direct comparison of the decomposed $\mathcal{S}$ and the WheelerKiladis diagrams highlights the tropical modes that play an important role in the equatorial momentum balance. We also translate the wavenumber-frequency information to the phase speeds of the disturbances for direct comparison with the zonal-mean zonal wind-an analysis that facilitates inspection for wave mean-flow interaction.

\section{Description of parameter end members}

To gain insight into how the nondimensional parameters change the atmospheric circulation we look at the climatological values of zonal-mean zonal winds, mass streamfunction, meridional eddy momentum flux $\overline{u^{\prime} v^{\prime}} \cos \phi$, and zonal-mean temperature for the end members of the parameter space. The results are averaged over the last 2200 days of 3000-day integrations. Since our experiments only change one nondimensional variable at a time, we label each figure by the nondimensional parameter that has been changed. For example, the $R_{o}=10$ figure refers to the experiment where $R_{o}$ has been changed and all other nondimensional parameters are held fixed at the control Earthlike value. Table 2 serves as a reference for all experiments.

\section{a. Control atmosphere}

First, the Earthlike control atmosphere is shown in Fig. 1. The control values of the nondimensional and dimensional parameters can be found in Table 2. The control atmosphere, like Earth's atmosphere, does not superrotate. There is a single jet in each hemisphere and easterlies at all equatorial pressure levels. The Hadley cells extend to $30^{\circ}$ and a depth of $200 \mathrm{hPa}$. Eddy activity is concentrated in midlatitudes and the tropics are decelerated by eddies in the mean; that is, $\mathcal{S}<0$ at the equator.

\section{b. Large thermal Rossby number}

The situation changes dramatically at large thermal Rossby number $\left(R_{o}=10\right.$, small radius $a=2.8 \times 10^{5} \mathrm{~m}$; Fig. 2). The bulk of the atmosphere is taken up by westerlies, with weak jets seen at $60^{\circ}$. The Hadley cell extends farther poleward, as expected from simple scaling arguments (Held and Hou 1980). The Hadley cell is also significantly shallower and weaker than that of the control atmosphere. The eddy activity has shifted equatorward, weakened, and moved to the lower troposphere. The eddies now accelerate the equatorial zonal winds, driving the superrotation. The temperature field is significantly flatter than the control since the "tropics," where the Rossby number is large, now extends to the poles (e.g., Sobel et al. 2001).

\section{c. Nondimensional radiative time scale}

With a small nondimensional radiative time scale $(\tau$ $=0.063$, strong Newtonian cooling $\tau_{r}=0.005$ days; Fig. $3)$, the Newtonian cooling term strongly restores temperatures to the forcing profile. As the forcing profile has its baroclinic zone much closer to the equator than the control climate (cf. Fig. 1c), the jets and Hadley cell are collapsed equatorward. The strict relaxation back to the forcing profile may also explain why the jet cores shift upward: in this strongly damped climate, the meridional temperature gradient no longer changes sign moving from the surface to the upper troposphere. Because radiative cooling balances heating by subsidence 


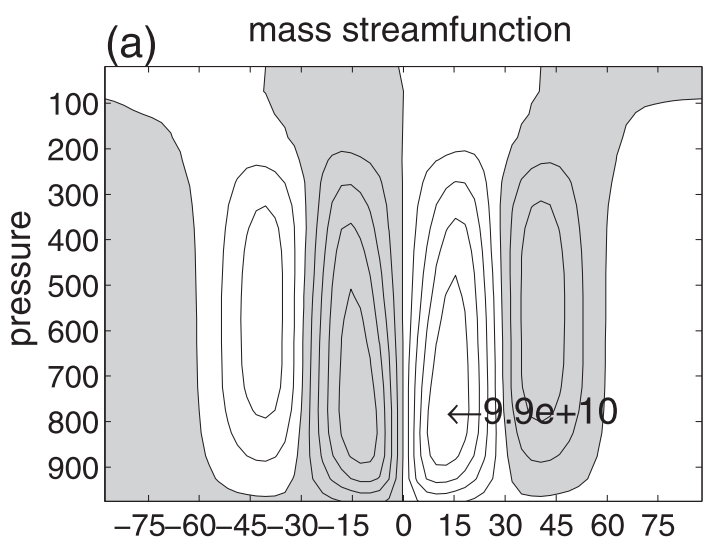

(c)

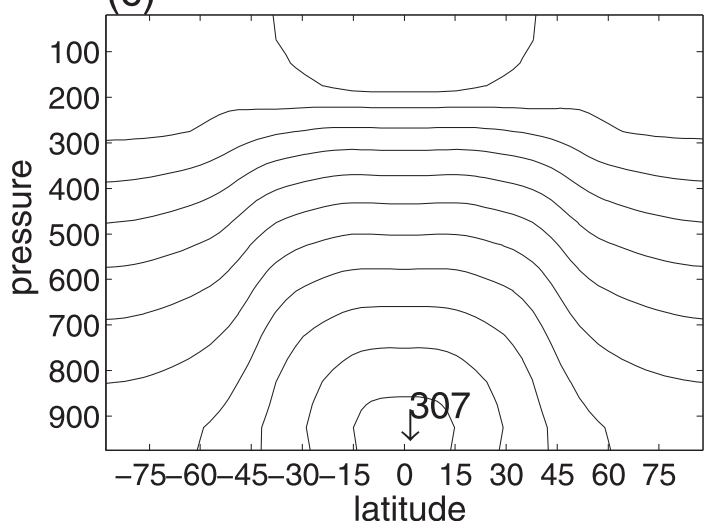

(b) zonal mean zonal wind

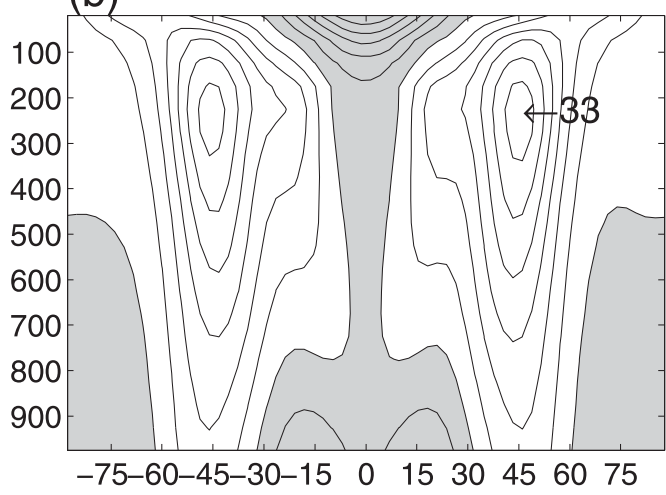

(d) meridional eddy momentum flux

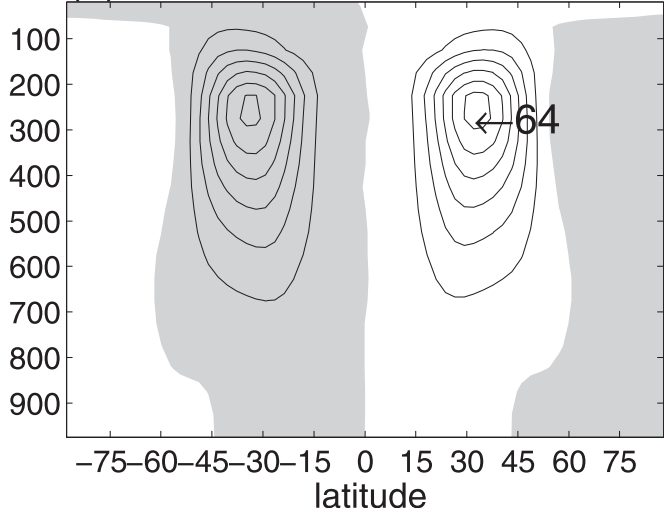

FIG. 1. Time-mean fields for the control atmosphere. (a) Mass streamfunction (contour interval $2 \times 10^{10} \mathrm{~kg} \mathrm{~s}^{-1}$ ). (b) Zonal-mean zonal winds (contour interval $5 \mathrm{~m} \mathrm{~s}^{-1}$ ). (c) Zonal-mean temperature (contour interval $10 \mathrm{~K}$ ).

(d) $\overline{u^{\prime} v^{\prime}} \cos \phi$ (contour interval $10 \mathrm{~m}^{2} \mathrm{~s}^{-2}$ ). Shaded areas are negative values. Largest values are highlighted.

in the Hadley cell's descending branch (Held and Hou 1980), strong diabatic cooling allows the Hadley cell to strengthen. Strong easterlies lie over the entirety of the tropics.

At large radiative time scale $(\tau=12600$, weak Newtonian cooling $\tau_{r}=1000$ days; Fig. 4), the Newtonian cooling term is much weaker. A meridionally confined Hadley circulation allows for a second thermally direct circulation at higher latitudes. A second (weak) jet and region of surface westerlies can be seen at higher latitudes. The eddy activity shrinks equatorward and becomes much weaker relative to the control.

\section{d. Ekman number}

At large Ekman number $(E=7.9$, strong friction $r=$ 100 days $^{-1}$; Fig. 5), weak westerlies lie over the equator from the middle to high troposphere, and eddy activity has been damped by the strong friction. The HeldSuarez frictional layer extending from 1000 to $700 \mathrm{hPa}$ exerts an enormous influence on the structure of the Hadley cell, which now expands to fill the entire domain.
The frictional layer can also be seen in the zonal-mean zonal winds with the "surface westerlies" appearing between 800 and $700 \mathrm{hPa}$. Above the boundary layer, however, the Hadley cell resembles the control case with a slightly weaker mass flux. The strong friction damps the effectiveness of eddy heat transport and the zonalmean temperature is not far removed from the Newtonian cooling profile.

At small Ekman number $\left(E=4.0 \times 10^{-4}\right.$, weak friction $r=0.005$ days $^{-1}$; Fig. 6$)$, extremely strong easterlies lie at the equator. The global winds have strengthened considerably, and the jets have moved upward and poleward. The eddy activity, however, is quite weak. The slanted Hadley Cell and eddy variability show that the atmosphere experiences strong symmetric instabilities.

\section{e. Nondimensional vertical temperature gradient}

An atmosphere with small vertical temperature forcing gradient $\left(\mu=0.0017\right.$, weak stratification $\Delta_{v}=0.1 \mathrm{~K}$; Fig. 7) has a Hadley cell that only reaches to $15^{\circ}$. The Hadley cell has strengthened by $50 \%$ over the control 

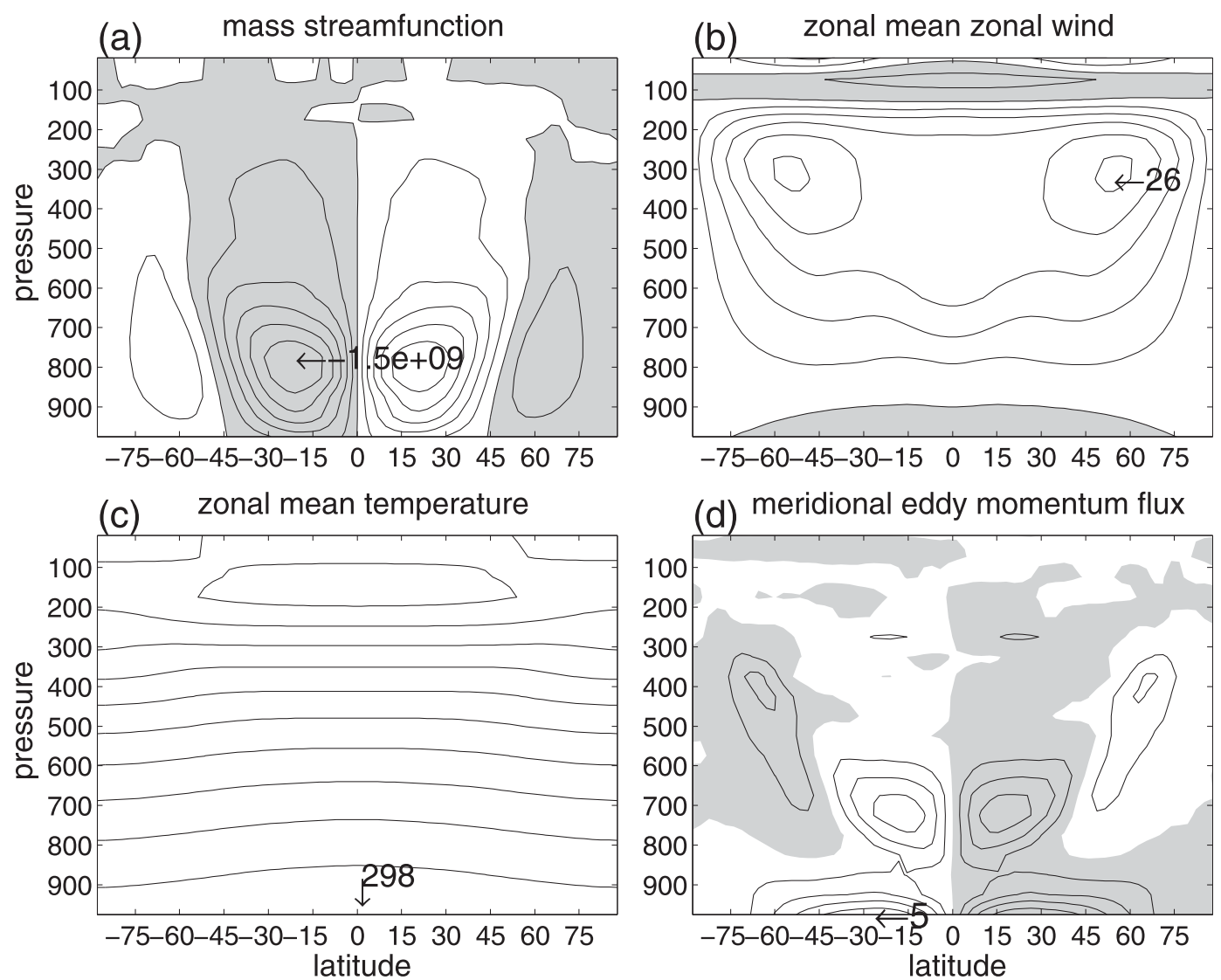

FIG. 2. Time-mean fields for the $R_{o}=10$ (small radius) atmosphere. (a) Mass streamfunction (contour interval $2.5 \times 10^{8} \mathrm{~kg} \mathrm{~s}^{-1}$ ). (b) Zonal-mean zonal winds (contour interval $5 \mathrm{~m} \mathrm{~s}^{-1}$ ). (c) Zonal-mean temperature (contour interval $10 \mathrm{~K})$. Shaded areas are negative values. (d) $\overline{u^{\prime} v^{\prime}} \cos \phi\left(\right.$ contour interval $1 \mathrm{~m}^{2} \mathrm{~s}^{-2}$ ). Largest values are highlighted.

atmosphere to compensate for weaker subsidence heating in its downward branch. The jets and eddy activity both move equatorward. In addition to eddy activity weakening, the characteristic zonal wavenumber increases from 6 in the control to 8 (not shown). This change in eddy length scale reflects the change in stratification and, consequently, the change in the deformation radius.

A large vertical temperature forcing gradient $(\mu=1.7$, strong stratification $\delta_{v}=100 \mathrm{~K}$; Fig. 8) has a more dramatic effect. There is modest equatorial superrotation at several pressure levels, although there is little indication of acceleration by horizontal eddy fluxes. The Hadley cell is weaker, wider, and taller than the control, while jets and eddy activity have strengthened and shifted poleward and upward. In contrast to above, the characteristic zonal wavenumber has decreased to 3, reflecting the strong stratification's effect on the deformation radius. The entire depth of the tropical atmosphere is significantly warmer and the stratosphere has disappeared.

\section{Superrotation parameter regime}

Figure 9 summarizes the model integrations in parameter space. Figure 9a plots the pressure-weighted vertical-, time-, and zonal-mean equatorial zonal wind versus the four nondimensional parameters discussed above. Figure $9 \mathrm{~b}$ plots the maximum (in the vertical) of the equatorial zonal-mean zonal wind.

The figure showing maximum equatorial winds is included for completeness, but we concentrate on superrotation in the vertical mean. To understand this, we manipulate the zonal momentum equation [see Eq. (1)] by decomposing fields into time means and deviations following, for example, Kraucunas and Hartmann (2005), to get

$$
\begin{aligned}
\frac{\partial[\bar{u}]}{\partial t}= & f[\bar{v}]-[\bar{v}] \frac{1}{a \cos \phi} \frac{\partial}{\partial \phi}([\bar{u}] \cos \phi)-[\bar{\omega}] \frac{\partial[\bar{u}]}{\partial p} \\
& -\frac{1}{a \cos ^{2} \phi} \frac{\partial}{\partial \phi}\left(\left[\overline{u^{\prime} v^{\prime}}\right] \cos ^{2} \phi\right)-\frac{\partial\left[\overline{u^{\prime} \omega^{\prime}}\right]}{\partial p}-\left[\overline{F_{x}}\right]
\end{aligned}
$$




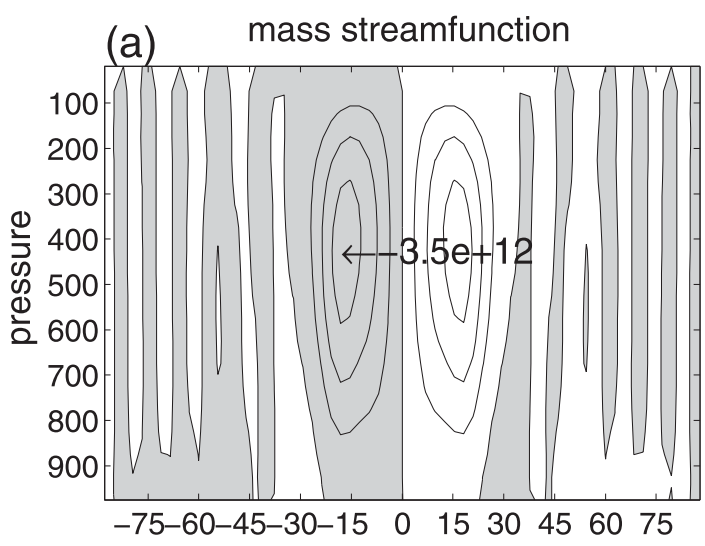

(c) zonal mean temperature

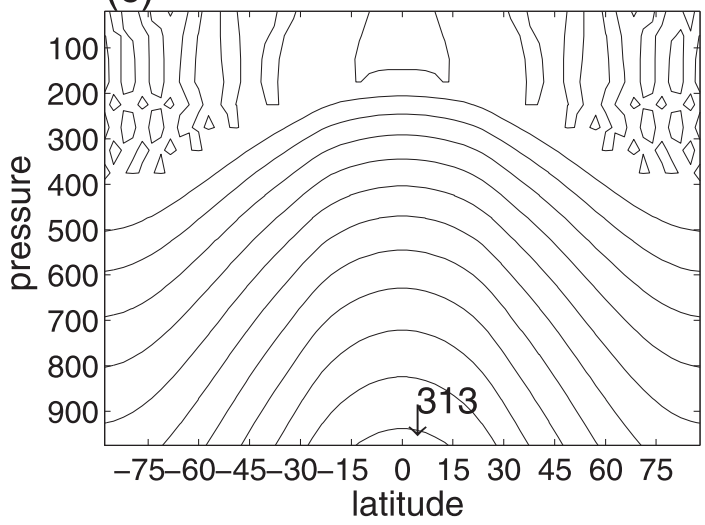

(b) zonal mean zonal wind

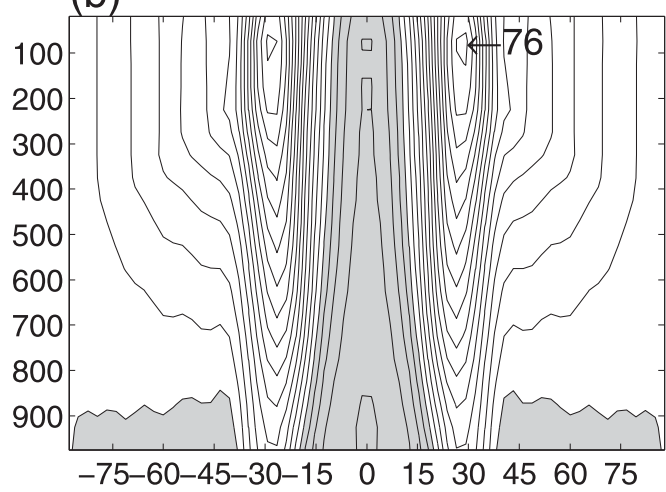

(d)

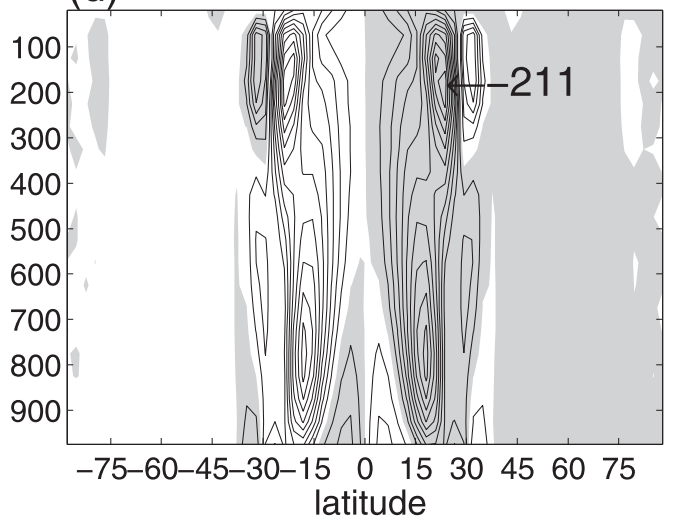

FIG. 3. Time-mean fields for the $\tau=0.063$ (strong Newtonian cooling) atmosphere. (a) Mass streamfunction (contour interval $1 \times 10^{12} \mathrm{~kg} \mathrm{~s}^{-1}$ ). (b) Zonal-mean zonal winds (contour interval $5 \mathrm{~m} \mathrm{~s}^{-1}$ ). (c) Zonal-mean temperature (contour interval $10 \mathrm{~K}$ ). (d) $\overline{u^{\prime} v^{\prime}} \cos \phi$ (contour interval $20 \mathrm{~m}^{2} \mathrm{~s}^{-2}$ ). Shaded areas are negative values. Largest values are highlighted.

where $F_{x}$ is the Rayleigh drag acting on the zonal flow. The metric terms and diffusion of horizontal momentum are neglected. The term $\left[\overline{u^{\prime} v^{\prime}}\right]$ is the transient meridional zonal momentum flux and $\left[\overline{u^{\prime} \omega^{\prime}}\right]$ is the transient vertical zonal momentum flux. As a note, these equations are only valid for axisymmetric forcing that is constant in time, and the inclusion of zonally asymmetric forcing would add stationary eddy terms of both meridional and vertical zonal momentum fluxes. Taking the pressure-weighted vertical mean (denoted by angle brackets) of Eq. (6) and using the zonal-mean mass conservation equation gives

$$
\begin{aligned}
\frac{\partial\langle[\bar{u}]\rangle}{\partial t}= & -\frac{1}{a \cos ^{2} \phi} \frac{\partial}{\partial \phi}\left(\langle[\bar{u}][\bar{v}]\rangle \cos ^{2} \phi\right) \\
& -\frac{1}{a \cos ^{2} \phi} \frac{\partial}{\partial \phi}\left(\left\langle\left[\overline{u^{\prime} v^{\prime}}\right]\right\rangle \cos ^{2} \phi\right)-\langle r[\bar{u}]\rangle .
\end{aligned}
$$

In the vertical mean, the Coriolis term disappears because of mass conservation - there cannot be a mean flow of mass out of the column. More importantly for our study, the vertical eddy momentum flux divergence term disappears since $\left[\overline{u^{\prime} \omega^{\prime}}\right]$ is zero at the surface and the top of the atmosphere. Combining Eq. (7) with Hide's theorem-a maximum in angular momentum must be maintained by eddy momentum transport-says that superrotation in the vertical mean must be maintained by meridional eddy momentum fluxes. Conversely, when superrotation exists at only a few model levels but the vertical-mean equatorial winds are easterly, the superrotation can be driven by either vertical or horizontal eddy momentum fluxes. This provides a way to distinguish between superrotation that is definitely [i.e., the quasi-biennial oscillation (QBO) and some models of Venus's superrotation; e.g., Fels and Lindzen 1974; Zhu 2006] or may possibly be (i.e., the small- $\tau$ runs; Fig. 3 ) driven by vertical eddy momentum fluxes and that which is definitely driven by meridional momentum fluxes (i.e., the large- $R_{o}$ runs; Fig. 2).

As an aside, Eq. (7) provides a fundamentally different view of the columnar momentum balance between 
(a)

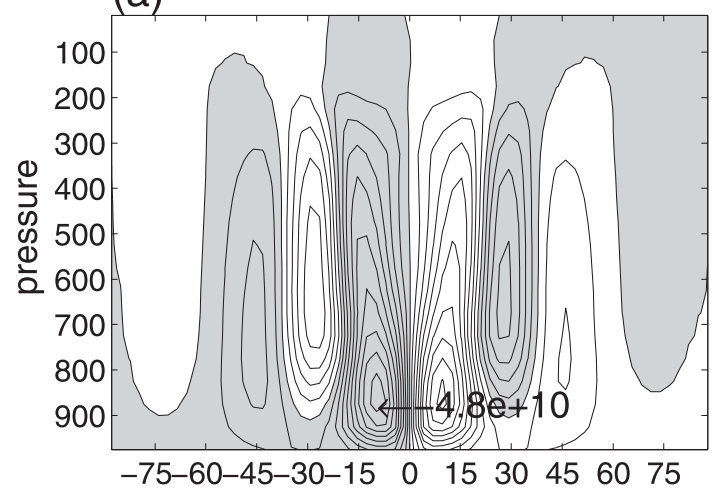

(c)

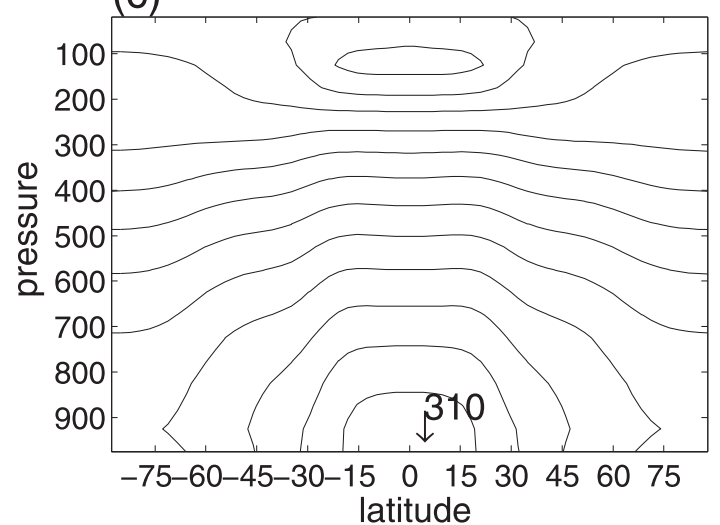

(b) zonal mean zonal wind

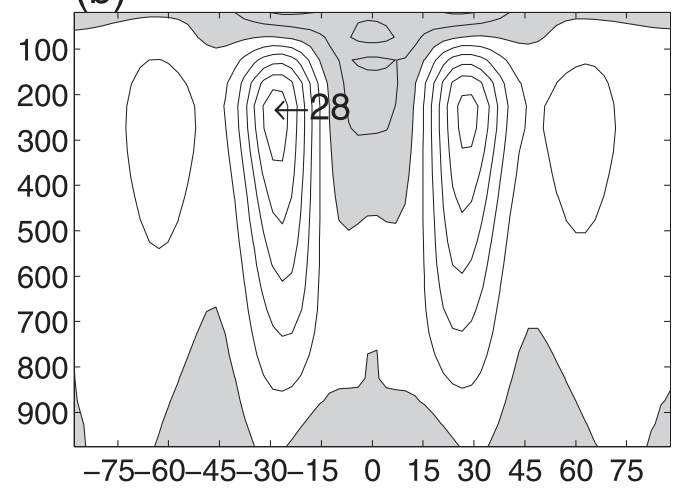

(d) meridional eddy momentum flux

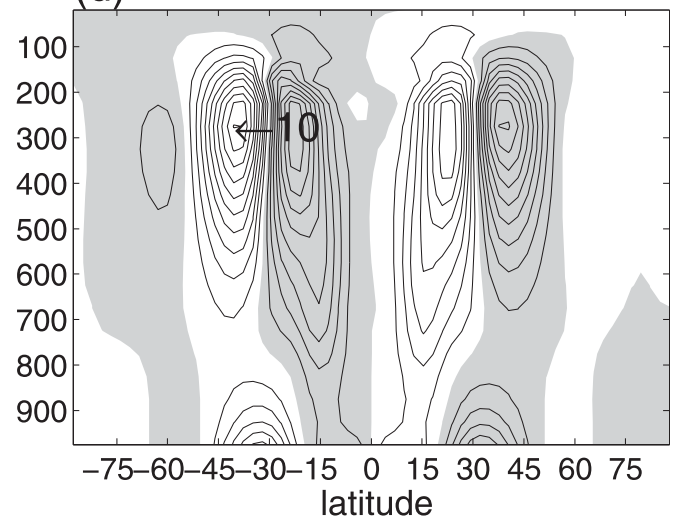

FIG. 4. Time-mean fields for the $\tau=12600$ (weak Newtonian cooling) atmosphere. (a) Mass streamfunction (contour interval $5 \times 10^{9} \mathrm{~kg} \mathrm{~s}^{-1}$ ). (b) Zonal-mean zonal winds (contour interval $5 \mathrm{~m} \mathrm{~s}^{-1}$ ). (c) Zonal-mean temperature (contour interval $10 \mathrm{~K}$ ). Shaded areas are negative values. (d) $\overline{u^{\prime} v^{\prime}} \cos \phi\left(\right.$ contour interval $\left.1 \mathrm{~m}^{2} \mathrm{~s}^{-2}\right)$. Largest values are highlighted.

the equator of a permanent-equinox superrotating atmosphere and the midlatitudes of an Earthlike atmosphere. In the midlatitudes of Earthlike atmospheres, Eq. (7) can be approximated as (in steady state) $\langle\mathcal{S}\rangle=\langle r[\bar{u}]\rangle$ (e.g., Schneider 2006). This balance can be interpreted as the need for surface westerlies to remove westerly momentum from the column in the same region where Rossby waves flux westerly momentum into the column. However, this balance is fundamentally different at the equator of a superrotating permanent-equinox atmosphere. The balance now requires another term and becomes $1 /\left(a \cos ^{2} \phi\right) \partial / \partial \phi\left(\langle[\bar{u}][\bar{v}]\rangle \cos ^{2} \phi\right)=\langle\mathcal{S}\rangle-\langle r[\bar{u}]\rangle$. As we have argued above, an atmosphere with westerlies in the equatorial vertical mean must have $\mathcal{S}>0$. Additionally, the Rayleigh drag term $-\langle r[\bar{u}]\rangle$ is also greater than zero because there are mean easterlies in the tropical boundary layer owing to the return flow of the Hadley cell. Therefore, both eddies and drag add momentum to the equatorial atmosphere and the Hadley cell takes it away.
Going back to Fig. 9a, vertical-mean equatorial westerlies only exist at large thermal Rossby number (squares), and so it is the dominant control parameter for superrotation driven by meridional momentum fluxes. Starting from an Earthlike atmosphere, for strong stability (large $\Delta_{v}$; plus signs), strong friction (circles), and weak radiative damping (triangles), the vertical-mean winds go to zero. On the other end of the parameter range, strong easterlies dominate at small nondimensional radiative time scale and Ekman number. A similar parameter sweep starting from a large-thermalRossby-number atmosphere rather than the Earthlike control will be reported on in a separate work by Dias Pinto and Mitchell (2013, manuscript submitted to Icarus).

The maximum equatorial zonal winds show less organized trends. For the nondimensional radiative time scale (triangles), for instance, the model produces superrotation in at least one pressure level on either side of the control run. Also for $\Delta_{v}$ (plus signs), 

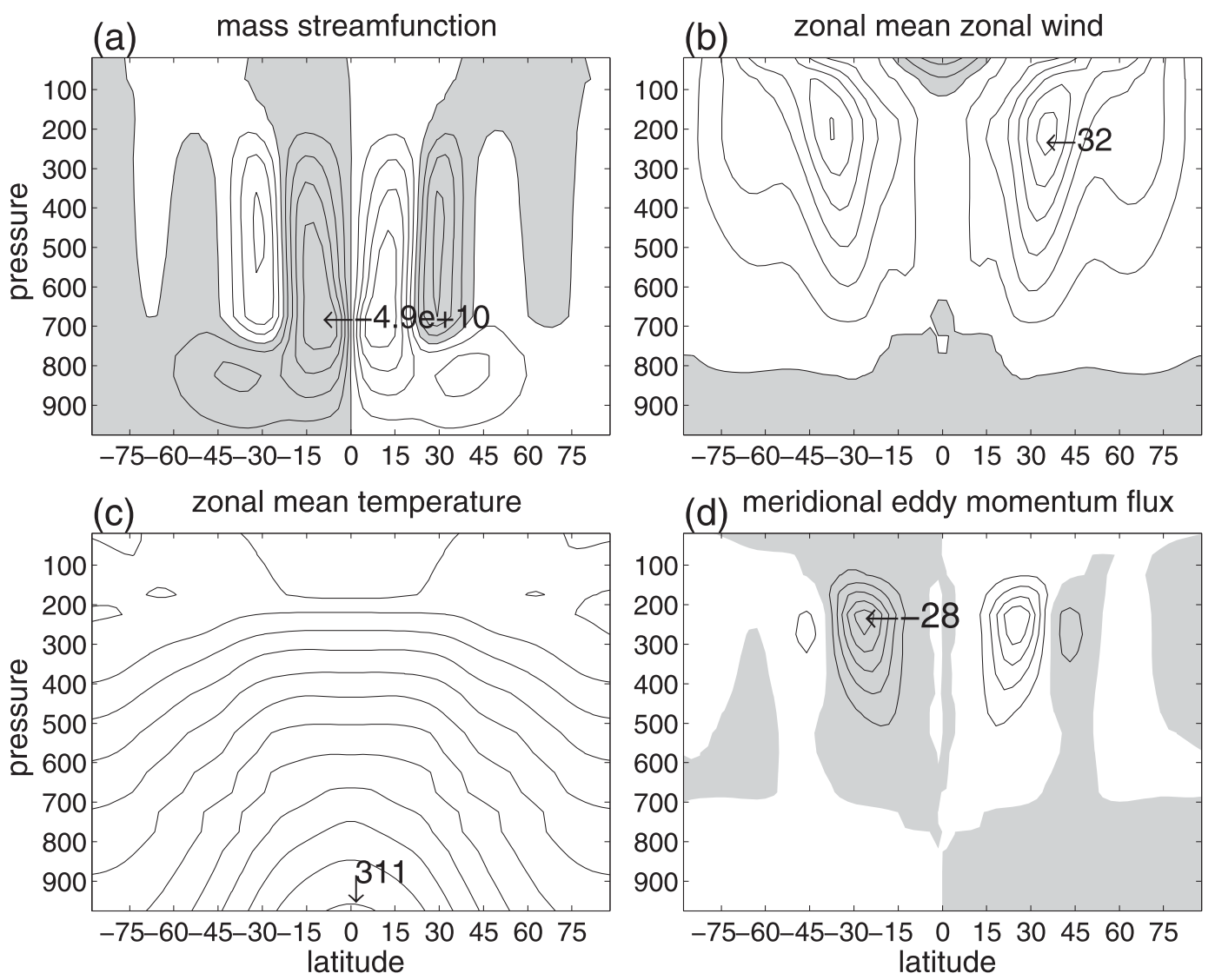

FIG. 5. Time-mean fields for the $E=7.9$ (strong friction) atmosphere. (a) Mass streamfunction (contour interval $1 \times 10^{10} \mathrm{~kg} \mathrm{~s}^{-1}$ ). (b) Zonal-mean zonal winds (contour interval $5 \mathrm{~m} \mathrm{~s}^{-1}$ ). (c) Zonal-mean temperature (contour interval $10 \mathrm{~K}$ ). (d) $\overline{u^{\prime} v^{\prime}} \cos \phi$ (contour interval $5 \mathrm{~m}^{2} \mathrm{~s}^{-2}$ ). Shaded areas are negative values. Largest values are highlighted.

there is strong superrotation at large values and there is very weak superrotation at small values. The maximum equatorial wind dependence of the thermal Rossby (squares) is similar to the dependency of the vertical mean, with westerlies existing for larger parameters.

Taken together, the most robust conclusion is that the thermal Rossby number is the dominant control parameter for equatorial superrotation.

\section{Spectral characteristics of the intermediate thermal Rossby number modes}

We now turn our focus to better understanding the variability driving the superrotation in the intermediate thermal Rossby number atmospheres. We focus on the $R_{o}=1\left(a=9 \times 10^{5} \mathrm{~m}\right)$ atmosphere since the $R_{o}=10$ $\left(a=2.8 \times 10^{5} \mathrm{~m}\right)$ atmosphere is less amenable to analysis and was explored in depth in MV10. Figure 10 shows climatological values for the $R_{o}=1$ atmosphere. The $R_{o}=1$ atmosphere superrotates more weakly than the
$R_{o}=10$ atmosphere (Fig. 2) but does contain somewhat stronger eddies. This likely reflects the fact that, unlike the $R_{o}=10$ atmosphere, the $R_{o}=1$ zonal-mean state (Fig. 10d) retains some of the control atmosphere's tendency for baroclinic instability.

We use the spectral methods of Wheeler and Kiladis (1999), Hayashi (1982), and Randel and Held (1991) to identify the tropical mode that gives rise to superrotation. A symmetric-mode Wheeler-Kiladis diagram of the geopotential height field at $275 \mathrm{hPa}$ for the control atmosphere is shown in Fig. 11a. Overlaid with solid lines are theoretical shallow-water dispersion curves for Kelvin waves and $n=1$ equatorial Rossby waves. These curves are drawn for equivalent depths $H=12,40$, and $200 \mathrm{~m}$. The clearest signal is the Kelvin wave corresponding to an equivalent depth of $40 \mathrm{~m}$. On the westward (left hand) side of the figure, the clearest signal is a global Rossby-Haurwitz wave (Hendon and Wheeler 2008; Kasahara 1980). The Rossby-Haurwitz dispersion relation can be approximately represented by the equatorial Rossby wave dispersion relation with a large 

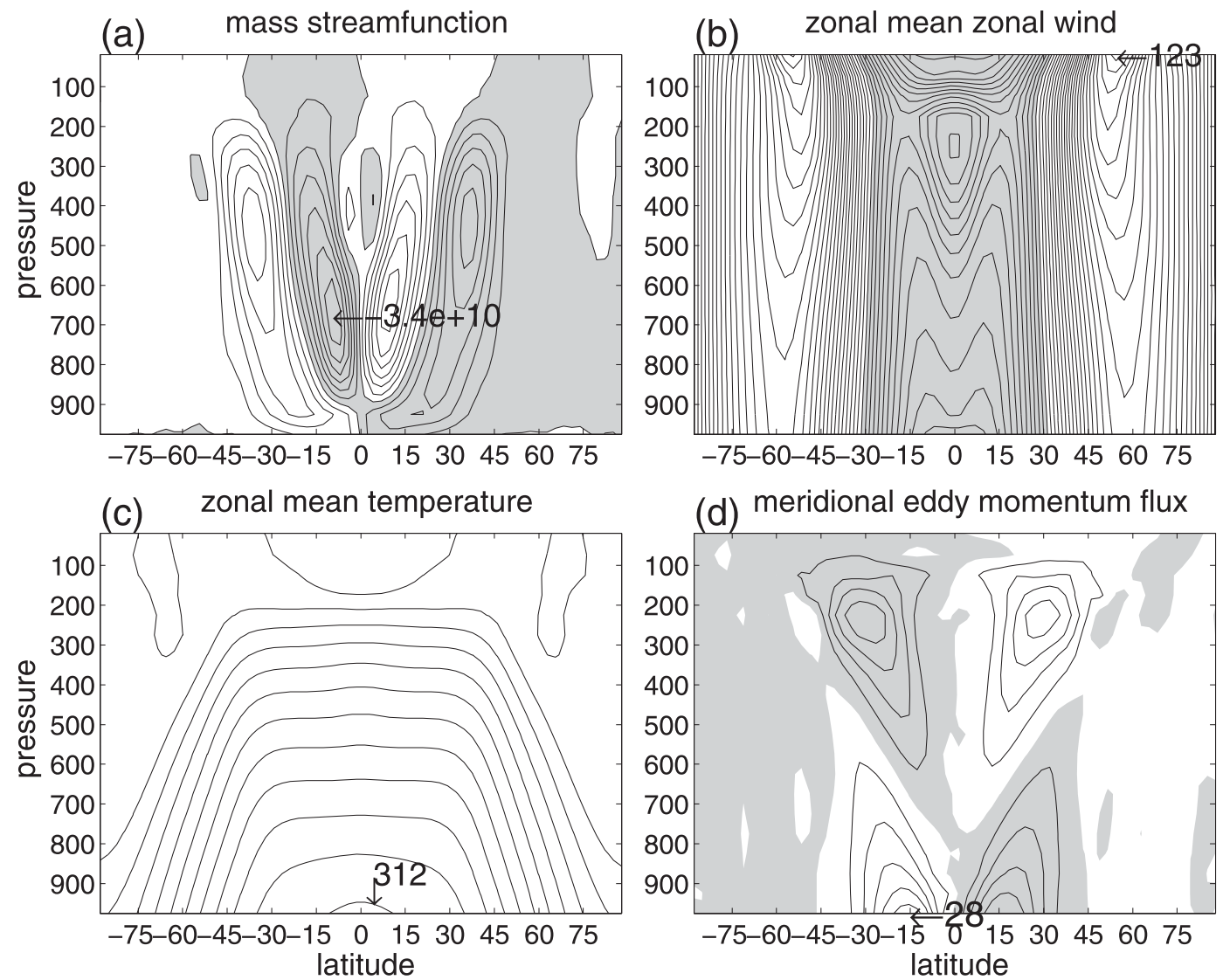

FIG. 6. Time-mean fields for the $E=4.0 \times 10^{-4}$ (weak friction) atmosphere. (a) Mass streamfunction (contour interval $5 \times 10^{9} \mathrm{~kg} \mathrm{~s}^{-1}$ ). (b) Zonal-mean zonal winds (contour interval $5 \mathrm{~m} \mathrm{~s}^{-1}$ ). (c) Zonal-mean temperature (contour interval $10 \mathrm{~K}$ ). (d) $\overline{u^{\prime} v^{\prime}} \cos \phi$ (contour interval $5 \mathrm{~m}^{2} \mathrm{~s}^{-2}$ ). Shaded areas are negative values. Largest values are highlighted.

equivalent depth and a Doppler-shifting wind (Hendon and Wheeler 2008). In this model an equivalent depth of $13 \mathrm{~km}$ and a Doppler-shifting wind of $15 \mathrm{~m} \mathrm{~s}^{-1}$ provide a good fit to the Rossby-Haurwitz mode. The large equivalent depth of the Rossby-Haurwitz mode is due to its barotropic structure.

How does this compare to the real atmosphere? Figure 12a is a symmetric-mode Wheeler-Kiladis diagram for brightness temperature from the Cloud Archive User Service (CLAUS) dataset (Robinson 2013). There are readily apparent differences. The strongest signal is the Madden-Julian oscillation at low frequencies and wavenumbers 1-5 (e.g., Wheeler and Kiladis 1999; Zhang 2005). A strong $n=1$ equatorial Rossby wave signal is apparent in the CLAUS data. It is not surprising that the dry model does not mimic real-world variability with any particular accuracy: the dry model has extremely idealized physics and, most importantly, no moist convection parameterization. Though the Rossby-Haurwitz mode does not show up when looking at brightness temperature, it is visible for Earth's atmosphere in dynamical fields like $u$ or geopotential height (Hendon and Wheeler 2008).

The Wheeler-Kiladis diagram for the geopotential height field at $475 \mathrm{hPa}$ in the $R_{o}=1\left(a=9 \times 10^{5} \mathrm{~m}\right)$ is shown in Fig. 13a. The level of the analysis is dropped from 275 to $475 \mathrm{hPa}$ to reflect the fact that eddy activity is lower in the high- $R_{o}$ atmospheres. Changing the pressure level of this analysis does not qualitatively change the results and Figs. 11a, 12a, and 13a are meant to be compared directly. Figure 13a plots frequencies up to $1 \mathrm{day}^{-1}$ to better capture the shift to higher-frequency variability when the radius is decreased. Figure 13a shows a much simpler picture than that of the control atmosphere. Almost all westward activity has vanished and the primary variability is wavenumber- 1 and -2 Kelvin waves. Note that the dispersion curves are steeper as $a$ has decreased. It is interesting that the dominant Kelvin wave variability in both the control and $R_{o}=1$ atmospheres lie along the 40-m-equivalentdepth line. In their paper, Wheeler and Kiladis (1999) noted that the background outgoing longwave radiation 

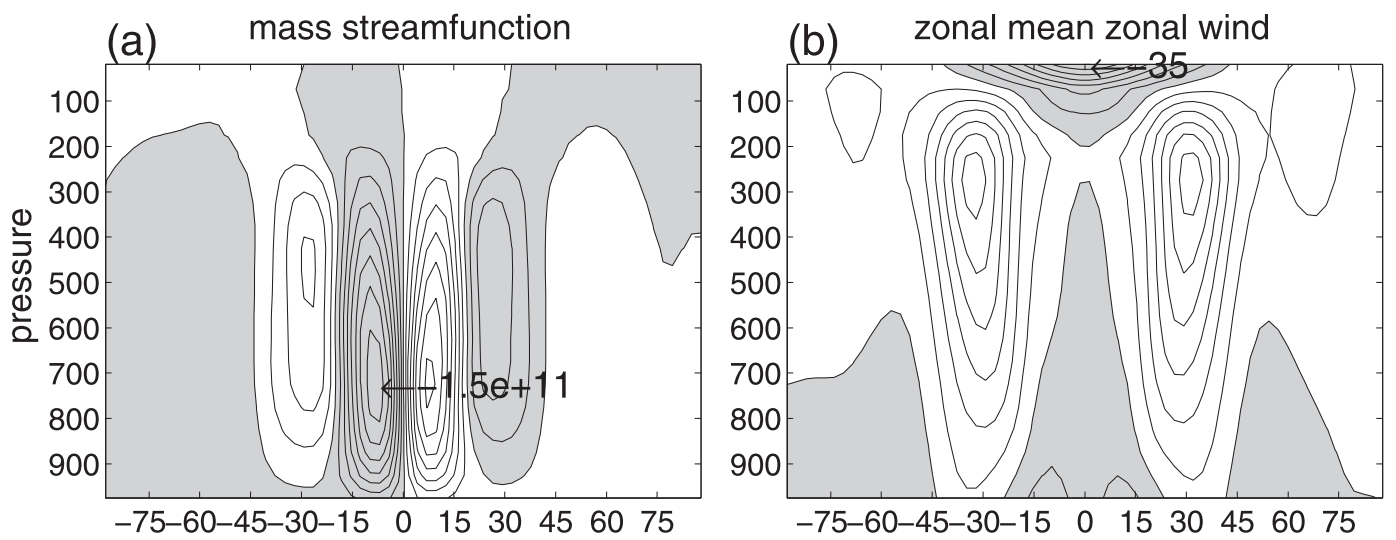

(c)

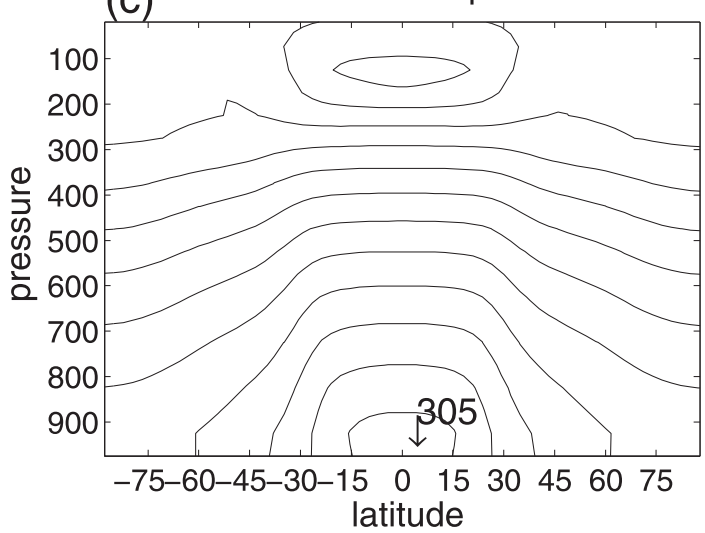

(d) meridional eddy momentum flux

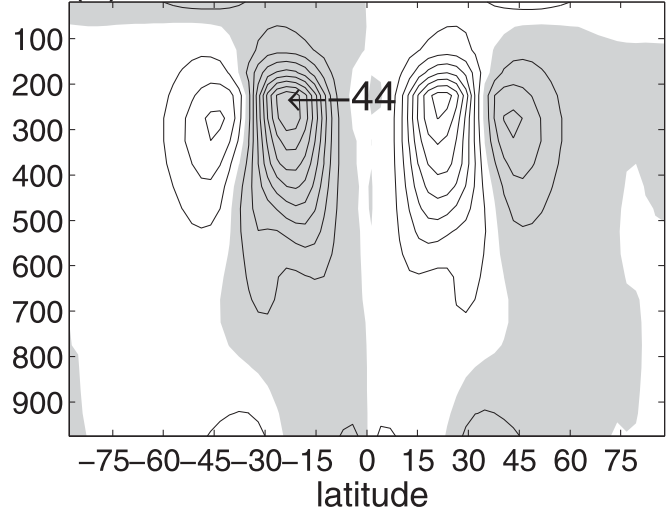

FIG. 7. Time-mean fields for the $\mu=0.0017$ (weak stratification) atmosphere. (a) Mass streamfunction (contour interval $2 \times 10^{10} \mathrm{~kg} \mathrm{~s}^{-1}$ ). (b) Zonal-mean zonal winds (contour interval $5 \mathrm{~m} \mathrm{~s}^{-1}$ ). (c) Zonal-mean temperature (contour interval $10 \mathrm{~K})$. (d) $\overline{u^{\prime} v^{\prime}} \cos \phi\left(\right.$ contour interval $5 \mathrm{~m}^{2} \mathrm{~s}^{-2}$ ). Shaded areas are negative values. Largest values are highlighted.

(OLR) spectrum is slightly weighted toward westward wavenumbers owing to the climatological easterlies overlying the equator. Assuming a half-wavelength structure in the troposphere (e.g., Kiladis et al. 2009) both the control and $R_{o}=1$ atmospheres have tropical equivalent depths of approximately $40 \mathrm{~m}$, which agrees with Figs. 11a and 13a. It is therefore somewhat surprising that the Kelvin wave variability in Fig. 13a lies along the 40-m-equivalent-depth line since any Kelvin wave would experience strong superrotating westerlies and would consequently be expected to be Doppler shifted to higher eastward frequencies. It is possible that the Kelvin wave has a steering level in the lower atmosphere where zonal winds are near zero but this needs to be investigated further.

While it is clear that the control and $R_{o}=1$ atmospheres have very different equatorial variability, it is unclear what is producing superrotation in the latter case. To answer this question, Figs. $11 \mathrm{~b}$ and $13 \mathrm{~b}$ plot equatorial $\mathcal{S}$, the transient eddy driving of zonal-mean zonal winds, in the same frequency-wavenumber space as the Wheeler-Kiladis diagrams discussed above (Hayashi 1982; Randel and Held 1991). This figure makes it possible to not only see which modes are present in the tropics but which are important for the tropical momentum balance. In Fig. 11b the control atmosphere $k>0$ eddies accelerate the zonal wind. This acceleration peaks at $k=1$ and extends at low frequency to higher wavenumbers. It is possible that this variability is due to low-frequency Kelvin waves. On the $k<0$ side of the spectrum, what appears to be a combination of Rossby waves propagating from midlatitudes and equatorial mixed Rossby-gravity waves decelerate the zonal wind. This stands in stark contrast to the eddy driving in the $R_{o}=1$ atmosphere, Fig. 13b, where the only significant features are at wavenumbers 1 and 2 . These features exactly correspond to the Kelvin waves highlighted in Fig. 13a.

As a side note, not too much should be read into the resemblance between the low-frequency $k>0$ variability in the control dry atmosphere (Fig. 11b) and the MJO in Earth's atmosphere (Fig. 12b). The point in the 

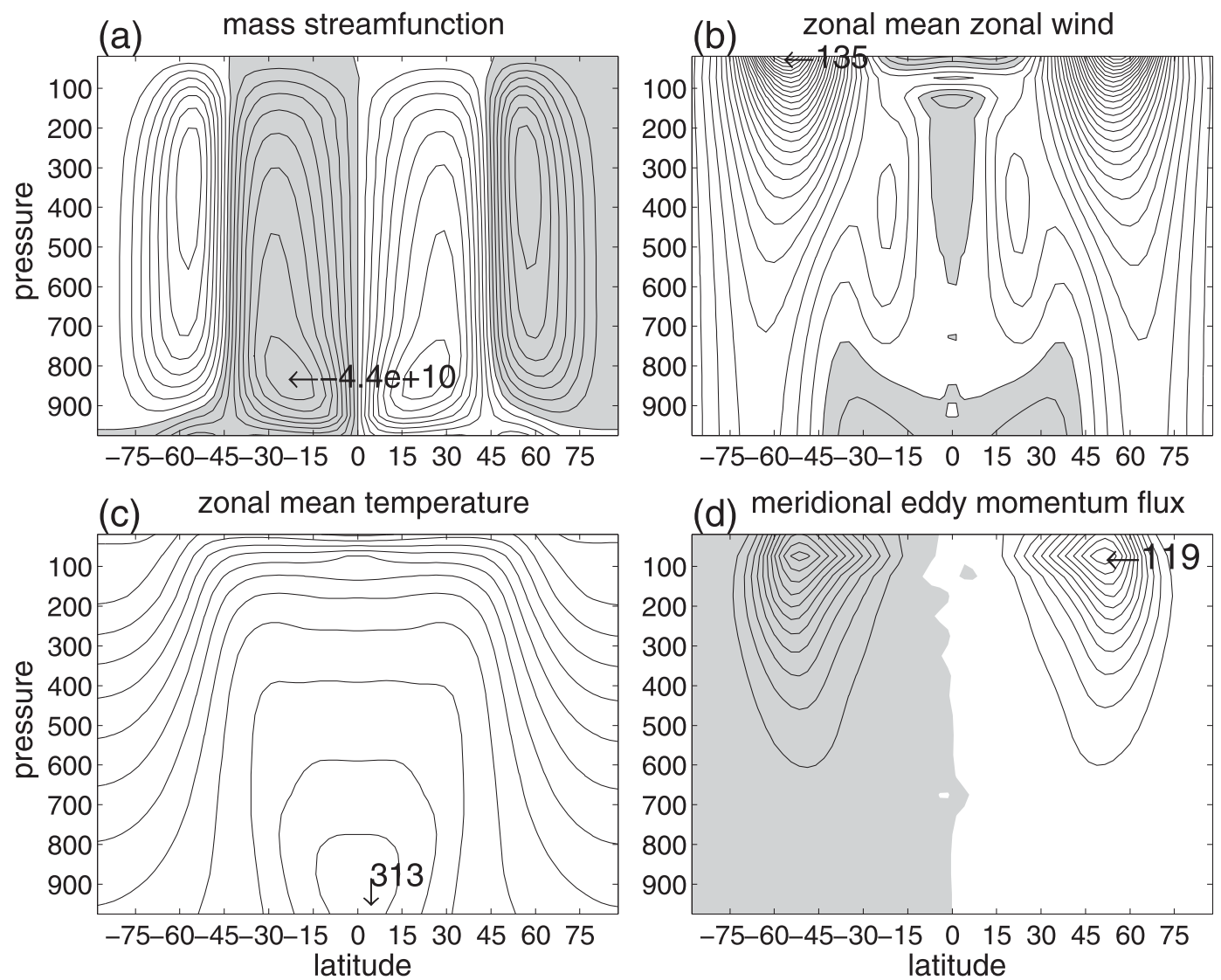

FIG. 8. Time-mean fields for the $\mu=1.7$ (strong stratification) atmosphere. (a) Mass streamfunction (contour interval $5 \times 10^{9} \mathrm{~kg} \mathrm{~s}^{-1}$ ). (b) Zonal-mean zonal winds (contour interval $5 \mathrm{~m} \mathrm{~s}^{-1}$ ). (c) Zonal-mean temperature (contour interval $10 \mathrm{~K}$ ). (d) $\overline{u^{\prime} v^{\prime}} \cos \phi$ (contour interval $10 \mathrm{~m}^{2} \mathrm{~s}^{-2}$ ). Shaded areas are negative values. Largest values are highlighted.

control atmosphere at which the Fig. 11b decomposition is done in the dry model, $275 \mathrm{hPa}$ at the equator, has in its mean state a meridional eddy momentum flux divergence of $-0.06 \mathrm{~m} \mathrm{~s}^{-1}$ day $^{-1}$. This is extremely weak variability when compared to the much larger acceleration of $1.8 \mathrm{~m} \mathrm{~s}^{-1} \mathrm{day}^{-1}$ at $475 \mathrm{hPa}$ at the equator for the $R_{o}=1$ atmosphere in Fig. 13, where the Kelvin wave signal is much stronger.

A plot of $\mathcal{S}$ as a function of phase speed and latitude is also shown in Fig. 14. The Kelvin wave's influence can be seen to extend $50^{\circ}$ from the equator, just beyond what appears to be a critical/turning line, where it draws momentum from the mean flow and deposits it at the equator. At higher latitudes, a separate mode is transporting momentum from even higher latitudes to the Kelvin wave's sink region. These modes combine in a "bucket brigade" to produce equatorial superrotation from the momentum of the high-latitude jets. The connection between the high- and low-latitude modes can also be seen in the dashed line. The dashed line shows phase speeds corresponding to a constant angular velocity that matches that of the peak Kelvin wave phase speed of $18 \mathrm{~m} \mathrm{~s}^{-1}$ at the equator. The acceleration due to the Kelvin wave is balanced by deceleration with the same angular velocity at higher latitudes.

It is not immediately obvious that a Kelvin wave can transport momentum meridionally since in the standard theory the wave does not manifest itself in the meridional velocity field: $v^{\prime}=0$ (Matsuno 1966). However, studies have shown the importance of Kelvin wave momentum fluxes in Venus-like models (Iga and Matsuda 2005; Imamura 2006) and Showman and Polvani (2011) showed that if phased correctly a Kelvin wave and an equatorial Rossby wave can produce equatorward momentum fluxes. Imamura et al. (2004) showed that in the presence of a meridional circulation that maintains meridional shear a Kelvin wave does have a small but important $v^{\prime}$ component that can lead to zonal accelerations. It is possible that this explains the momentum fluxes found in the $R_{o}=1$ atmosphere as an inspection 

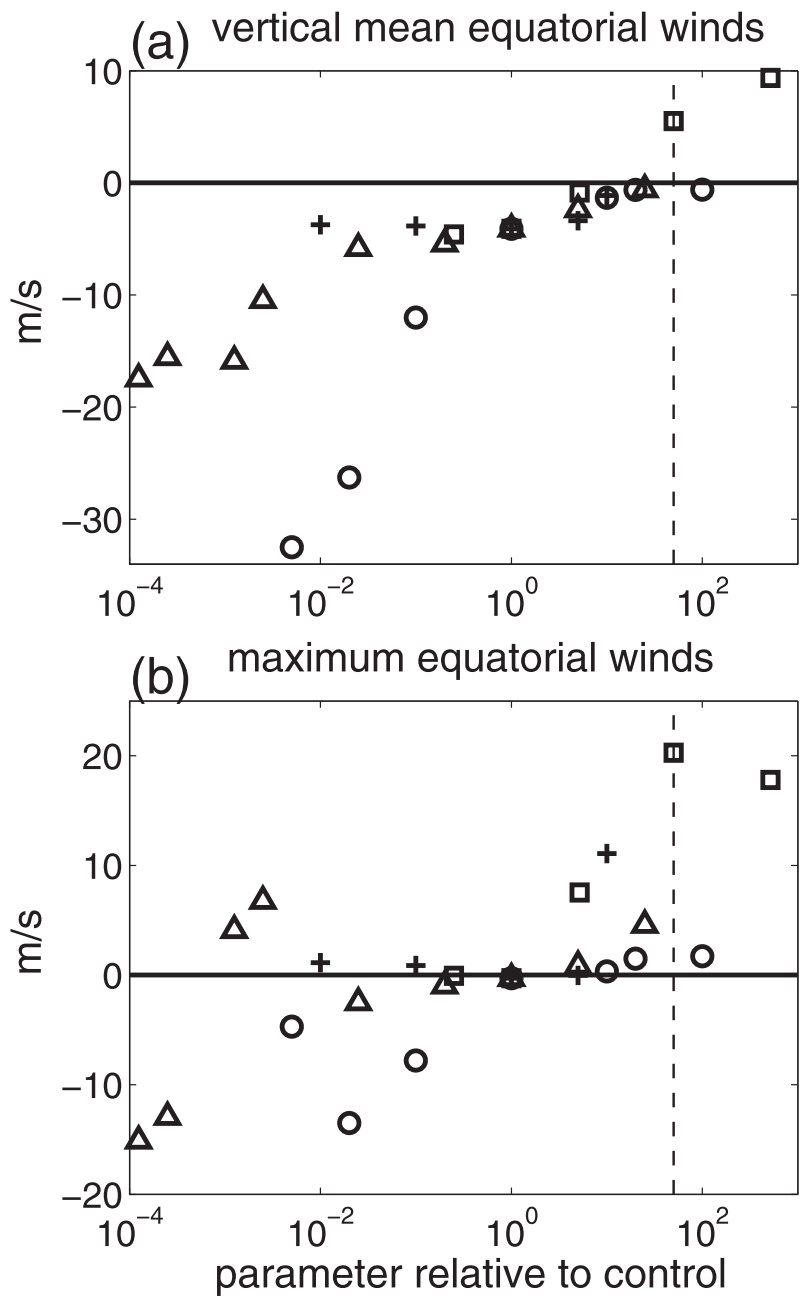

FIG. 9. (a) Vertical-mean equatorial winds and (b) maximum equatorial winds as a function of nondimensional parameters. Squares indicate an experiment with the thermal Rossby number varied, triangles represent the nondimensional radiative time scale, circles represent the Ekman number, and plus signs represent the nondimensional vertical temperature gradient. Nondimensional parameters are normalized by their control value. As a reference, the vertical dashed line denotes the location of the $R_{o}=1$ (50 times larger than the control $R_{o}=0.02$ ) case, which is discussed in detail in the text.

of Fig. 10 shows that the deep tropical momentum fluxes are collocated with the outflow of the Hadley cell. The specific structure of this Kelvin wave does need to be understood in greater detail, but we can conclude that the superrotation in the intermediate-thermal-Rossbynumber atmospheres is a direct result of Kelvin waves fluxing momentum meridionally into the deep tropics.

Further proof of the relevance of Kelvin wave meridional momentum transport in intermediate-thermalRossby-number atmospheres is demonstrated in Fig. 15. $\mathcal{S}$ as a function of wavenumber and frequency is shown at the equator for the $R_{o}=0.1\left(a=2.8 \times 10^{6} \mathrm{~m}\right)$ and $R_{o}=0.55\left(a=1.2 \times 10^{6} \mathrm{~m}\right)$ atmospheres. Eastward accelerations can be seen lying along the $H=40 \mathrm{~m}$ Kelvin wave dispersion curve.

\section{Wavenumber-frequency filtering}

To provide further evidence of the dry dynamical core's Kelvin wave, we use wavenumber-frequency filtering to show the structure of the model's primary modes (e.g., Straub and Kiladis 2003). A time series of zonal wind at $475 \mathrm{hPa}$ and a single equatorial latitude is Fourier transformed in time and space and filtered to only retain variability that exists for $k=1$ and frequencies between 0.17 and $0.37 \mathrm{day}^{-1}$. This filtered variability is inverse Fourier transformed to provide a time series that is regressed against $u, v$, and geopotential height. This process gives Fig. 16, which shows that the equatorial variability in the $R_{o}=1$ atmosphere is primarily due to a wavenumber- 1 Kelvin wave and the high-latitude variability is dominated by a wavenumber-1 Rossby wave. The equatorial part of Fig. 16 should be directly compared to the classical Kelvin wave solution of a beta-plane shallow-water model such as Fig. 3f of Kiladis et al. (2009). The connection between the Kelvin and Rossby waves seen here also corresponds to the acceleration and deceleration that fall along the same angular velocity curve in Fig. 14.

\section{Meridional Kelvin wave momentum transport in other contexts}

\section{a. Kelvin waves in Williams (2003)}

Further proof of the importance of Kelvin waves to the tropical momentum balance of dry dynamical cores is found by analyzing the results of Williams (2003). As discussed in the introduction, Williams induced superrotation in a dry dynamical core very similar to the one used here by changing the power in the latitudinal distribution of thermal forcing from $\cos ^{2} \phi$ to $\cos ^{n} \phi$. The zonal-mean zonal winds for a recreation of Williams's $n=16$ results is shown in Fig. 17a and should be compared to Fig. 5a in Williams (2003). Correspondence is not exact owing to slight differences in the models' details. As done before, the dynamically relevant tropical modes are identified by plotting $\mathcal{S}$ in $\omega-k$ space in Fig. 17b. The most prominent signal lies along the equivalent depth of 40-m Kelvin wave dispersion curve, though there are other features at low frequency and $\pm 5 k$. This result shows the relevance of Kelvin waves even in more Earthlike superrotating atmospheres. 

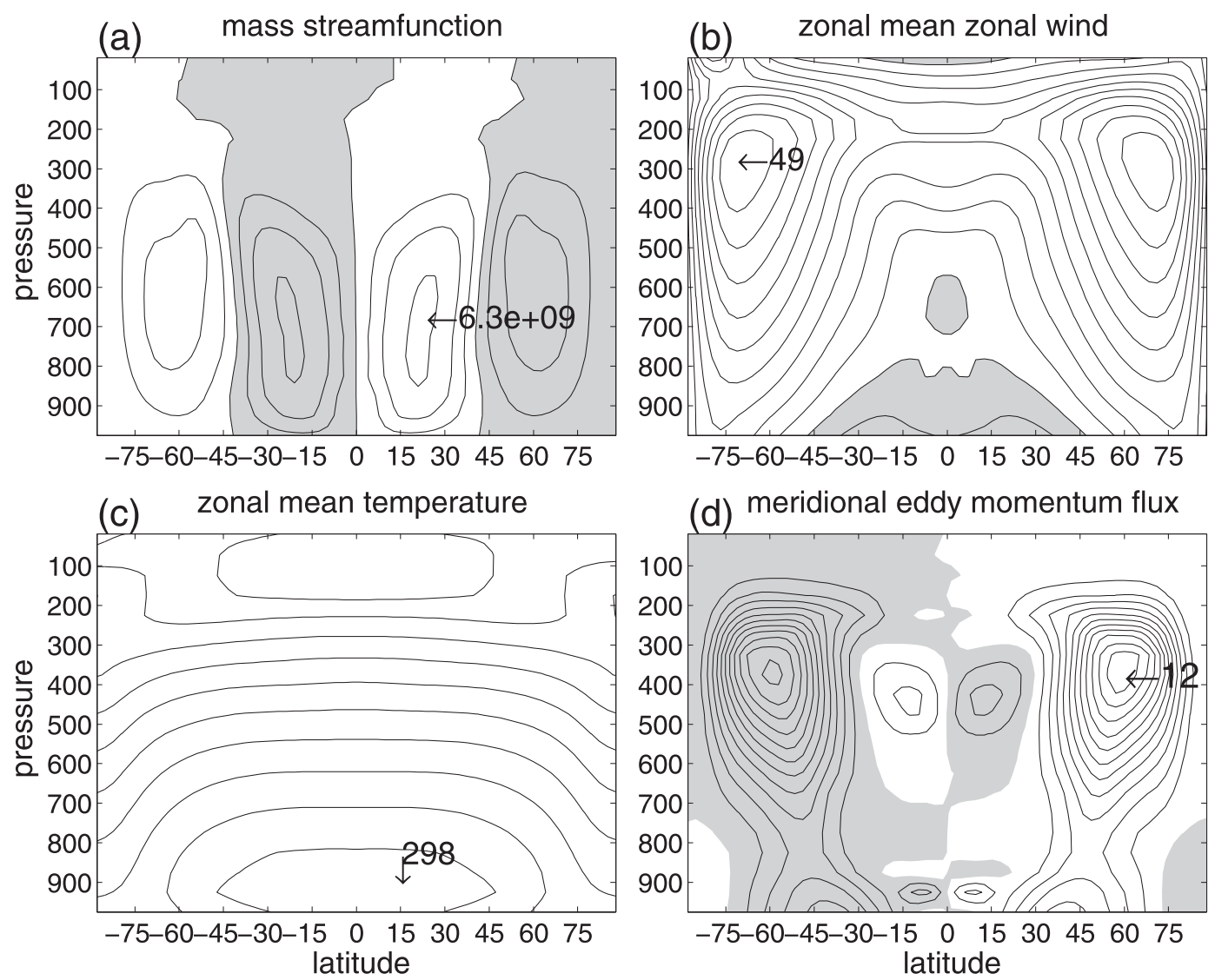

FIG. 10. Time-mean fields for the $R_{o}=1$ atmosphere. (a) Mass streamfunction (contour interval $2 \times 10^{9} \mathrm{~kg} \mathrm{~s}^{-1}$ ). (b) Zonal-mean zonal winds (contour interval $5 \mathrm{~m} \mathrm{~s}^{-1}$ ). (c) Zonal-mean temperature (contour interval $10 \mathrm{~K}$ ).

(d) $\overline{u^{\prime} v^{\prime}} \cos \phi$ (contour interval $1 \mathrm{~m}^{2} \mathrm{~s}^{-2}$ ). Shaded areas are negative values. Largest values are highlighted.

\section{b. Momentum flux divergence on Earth}

There is some correspondence between the dry dynamical core results and Earth's atmosphere. Figure 12 b plots $\mathcal{S}$ in $\omega-k$ space for Earth's atmosphere using the daily averaged 200-hPa National Centers for Environmental Prediction-U.S. Department of Energy (NCEP-DOE) Reanalysis II values of $u$ and $v$. The strongest signal in the NCEP diagram is at wavenumber 1 and low frequencies. This overlaps with the MJO signal in Fig. 12a and seems to imply that the strongest source of meridional eddy momentum flux in the upper-equatorial troposphere is associated with the MJO-a result previously shown by Lee (1999). Though the MJO seems to be the largest signal, the Kelvin wave meridional momentum convergence found in the dynamical core also exists in Earth's atmosphere. A clear Kelvin wave signal exists between wavenumbers 1 and 5. In the NCEP Reanalysis II the Hadley outflow peaks at $200 \mathrm{hPa}$, which may explain the clear Kelvin wave signal in Fig. 12b. At other tropospheric pressure levels, the Kelvin signal is weaker while the low-frequency, wavenumber-1 signal dominates. This fits within the theory of Imamura et al. (2004) that Kelvin wave momentum flux convergence is reliant on a meridional circulation.

\section{Discussion and conclusions}

In this paper, we have swept through a broad section of the parameter space of a nonconvecting dry dynamical core, thermally forced with a Newtonian relaxation, and found that the thermal Rossby number is the most important single nondimensional parameter governing the momentum balance of the equatorial troposphere. A high thermal Rossby number is, as in MV10, associated with a superrotating state. Of the other nondimensional quantities, a small Ekman number leads to strong equatorial easterlies, but a large Ekman number only induces weak superrotation at several model levels and not in the vertical mean. As the radiative time scale is decreased, the mean equatorial zonal winds become 
(a)

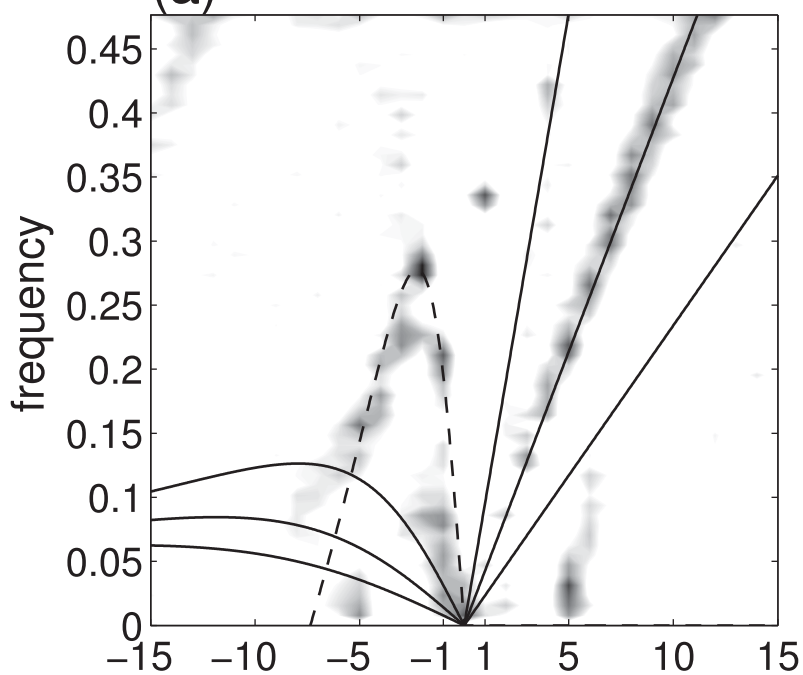

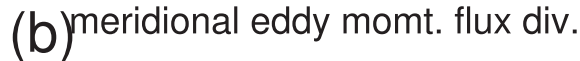

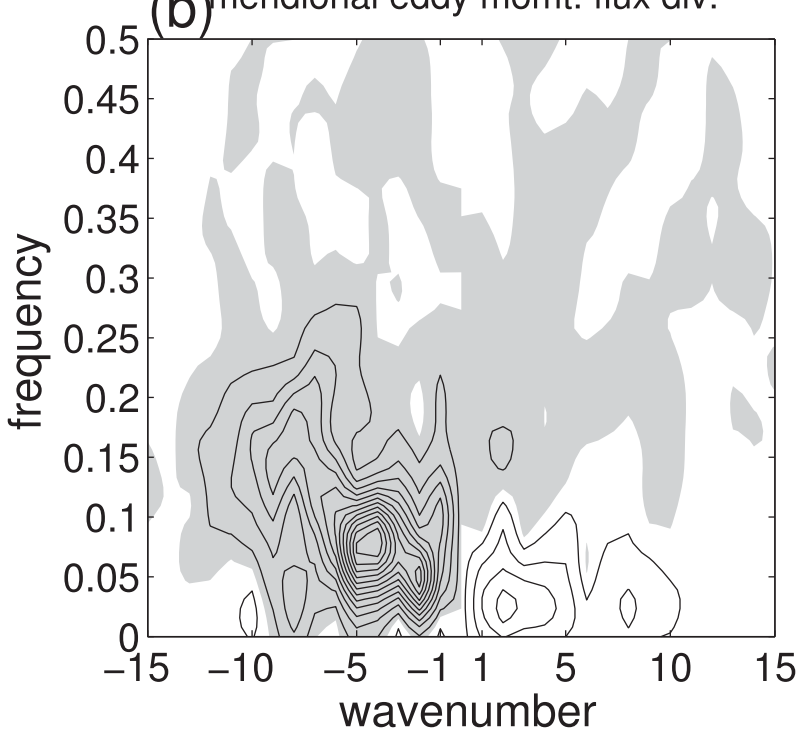

FIG. 11. Spectral diagnostics for the control atmosphere at $275 \mathrm{hPa}$. (a) Symmetric-mode Wheeler-Kiladis diagram of the geopotential height field. Maximum amplitude is 2.4. Solid curves show theoretical dispersion relations for Kelvin waves $(k>0)$ and equatorial Rossby waves $(k<0)$ of equivalent depths $H=200,40$, and $12 \mathrm{~m}$. Dashed curve shows theoretical dispersion relation for a Rossby-Haurwitz wave with equivalent depth of $13 \mathrm{~km}$ Doppler shifted by a $15 \mathrm{~m} \mathrm{~s}^{-1}$ wind. (b) $\mathcal{S}$ in $\omega-k$ space at the equator at $275 \mathrm{hPa}$. Shaded values are negative. Contour intervals are arbitrary.

strongly easterly, and as the time scale is increased, these winds go to zero. Changing the radiative time scale or the vertical temperature forcing gradient can induce strong superrotation but only at a few pressure levels and not in the vertical mean.

The consequence of these results is that, at least in an atmosphere where latent heat release does not play an important role, superrotation will be a more likely

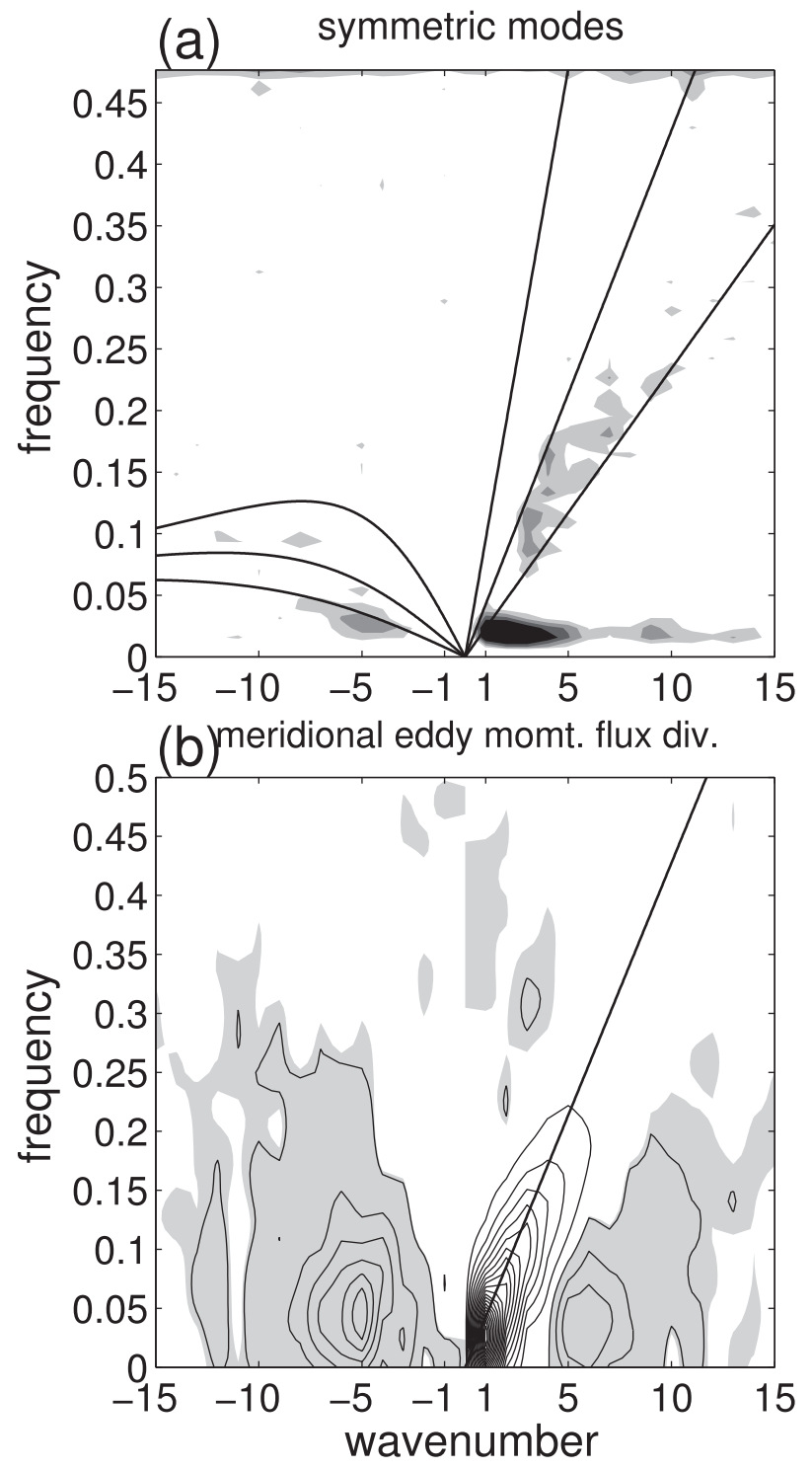

FIG. 12. Spectral diagnostics for Earth's atmosphere. (a) Symmetric-mode Wheeler-Kiladis diagram for the CLAUS brightness temperature dataset. Maximum amplitude is 1.9. Solid curves show theoretical dispersion relations for Kelvin waves $(k>0)$ and equatorial Rossby waves $(k<0)$ of equivalent depths $H=200,40$, and $12 \mathrm{~m}$. (b) $\mathcal{S}$ in $\omega-k$ space at the equator and $200 \mathrm{hPa}$ for the NCEP Reanalysis II. Solid line shows the $H=40 \mathrm{~m}$ Kelvin wave dispersion relation. Shaded values are negative. Contour intervals are arbitrary.

atmospheric state when the thermal Rossby number is large. Building on Caballero and Huber (2010) and Lee (1999), when latent heat release is important and organized convection leads to tropical phenomena such as the Madden-Julian oscillation and moist Kelvin modes, a warm and moist atmosphere with large thermal Rossby number is even more likely to superrotate than a dry atmosphere with the same large thermal Rossby 

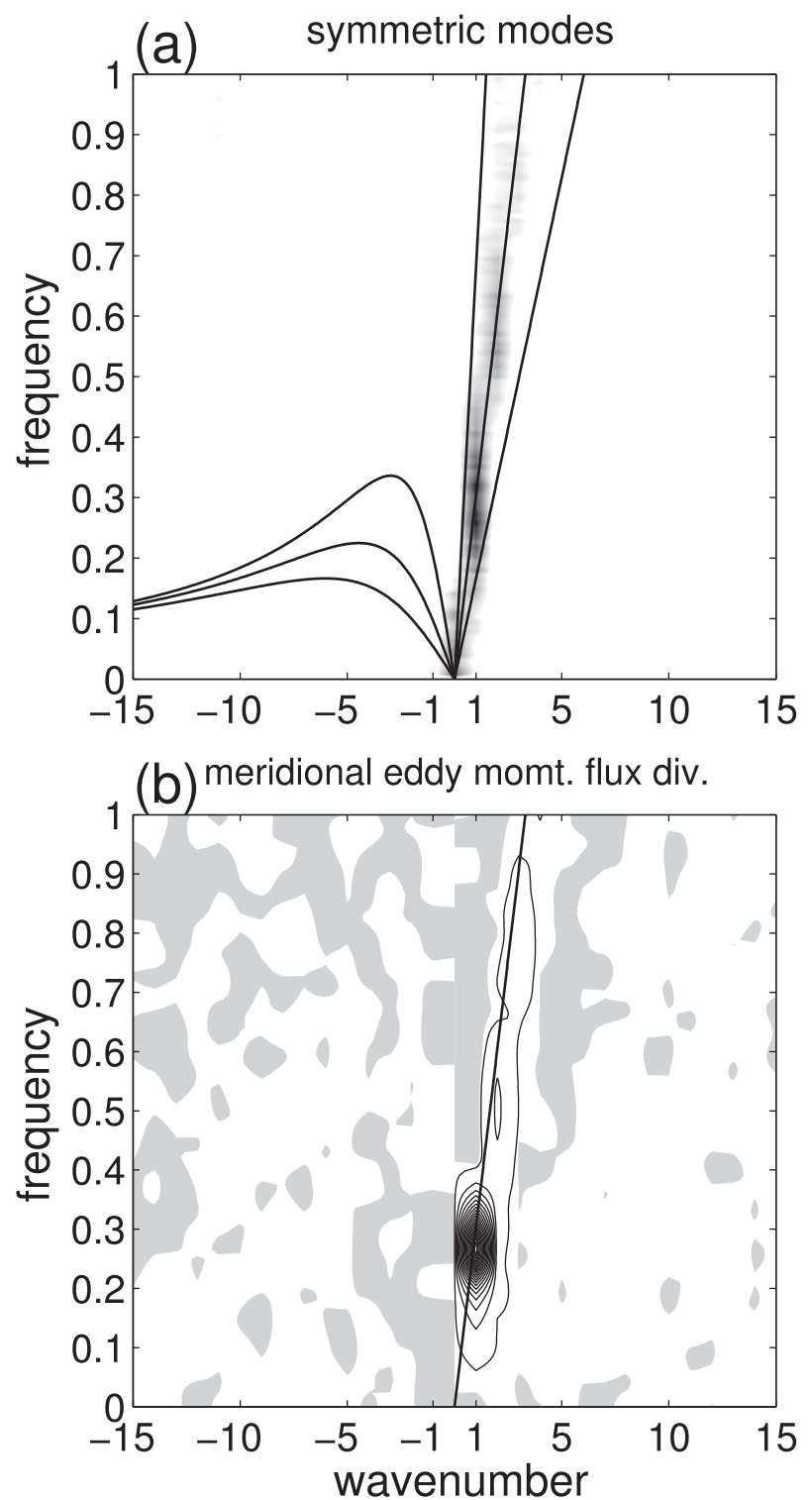

FIG. 13. Spectral diagnostics for the $R_{o}=1$ atmosphere at $475 \mathrm{hPa}$. (a) Symmetric-mode Wheeler-Kiladis diagram of the geopotential height field. Maximum amplitude is 5.8. Solid curves show theoretical dispersion relations for Kelvin waves $(k>0)$ and equatorial Rossby waves $(k<0)$ of equivalent depths $H=200$, 40 , and $12 \mathrm{~m}$. (b) $\mathcal{S}$ in $\omega-k$ space at the equator. Solid line shows the $H=40 \mathrm{~m}$ Kelvin wave dispersion relation. Shaded values are negative. Contour intervals are arbitrary.

number. Lian and Showman (2010) and Liu and Schneider (2011) have shown that convection plays a key role in generating superrotation on planets with larger radii, although it is unclear how their findings relate to our largethermal-Rossby-number (small radius) results.

Our parameter sweep results correspond with what is known of terrestrial planets with large-thermal-Rossbynumber atmospheres like Venus and Titan, both of which

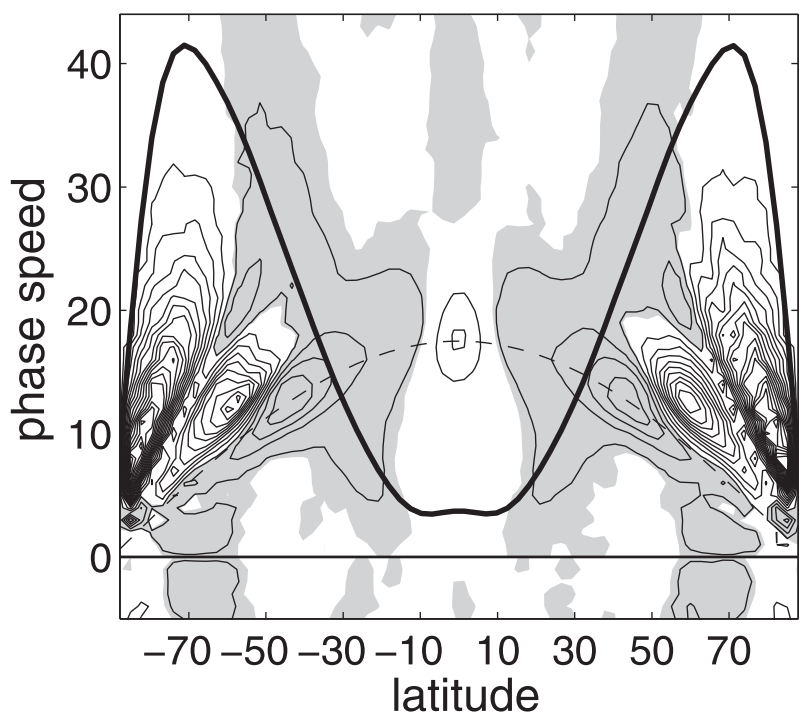

FIG. 14. Contours of $\mathcal{S}$ as a function of phase speed and latitude at $475 \mathrm{hPa}$ for the $R_{O}=1$ atmosphere. Dark line shows the time-mean zonal-mean zonal wind at the same pressure level. Shaded values are negative. Contour intervals are arbitrary. The dashed line shows phase speeds that correspond to the angular velocity that gives the peak Kelvin wave phase speed of $18 \mathrm{~m} \mathrm{~s}^{-1}$ at the equator.

are superrotating. However, it turns out that the superrotation at least on Titan is more subtle than simply having a large thermal Rossby number. Dias Pinto and Mitchell (2013, manuscript submitted to Icarus) have shown that the thermal damping time scale also plays an important role and when the overturning circulation is made to be too strong, superrotation can be shut down.

We have analyzed the dry dynamical core's tropical variability in an attempt to isolate the mechanism producing the superrotation-in particular, by decomposing various fields in spectral space and trying to identify the variability with wave motion. Using the uppertropospheric geopotential height field, we found that the control run of the dynamical core has strong Kelvin wave and Rossby-Haurwitz signals. Earth's atmosphere has richer variability, with strong Kelvin wave, equatorial Rossby wave, and MJO activity (focusing only on symmetric and lower-frequency modes). As the thermal Rossby number increases in the model, the Kelvin wave response grows stronger and low wavenumbers become dominant.

In particular, we decomposed the equatorial eddy momentum flux divergence $\mathcal{S}$ in spectral space and found that in the control atmosphere there exists a weak acceleration by eastward eddies (likely low-frequency Kelvin waves) that is overpowered by westward eddies (Rossby and mixed Rossby-gravity waves) decelerating the flow. The eddy activity changes as the thermal Rossby number increases and now equatorial eddies, 

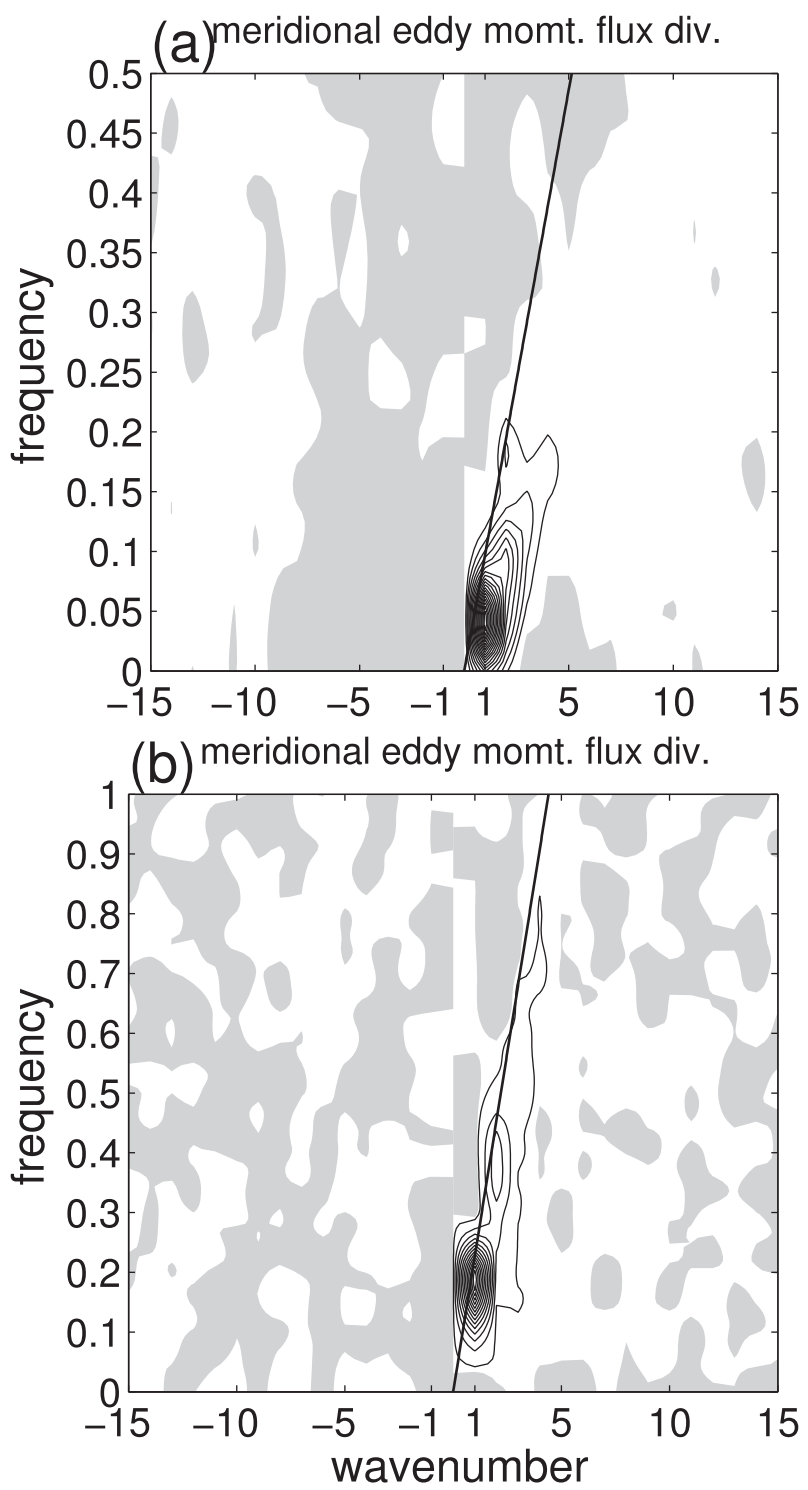

FIG. 15. (a) $\mathcal{S}$ in $\omega-k$ space at the equator for (a) the $R_{o}=0.1$ atmosphere at $275 \mathrm{hPa}$ and (b) the $R_{o}=0.55$ atmosphere at $425 \mathrm{hPa}$. Solid line shows the $H=40 \mathrm{~m}$ Kelvin wave dispersion relation. Shaded values are negative. Contour intervals are arbitrary.

with a Kelvin wave signature, strongly accelerate the equatorial zonal winds. Kelvin wave meridional momentum transport was shown to exist at other intermediate thermal Rossby numbers and Kelvin wave meridional momentum transport is also present in the superrotating cases of Williams (2003), despite the very different configuration.

Interestingly, Earth's atmosphere is also found to have strong eddy acceleration at the equator that corresponds to both the wavenumber- 1 component of the $\mathrm{MJO}$ and low-wavenumber Kelvin waves. The fact that superrotation does not occur in the Earth's atmosphere

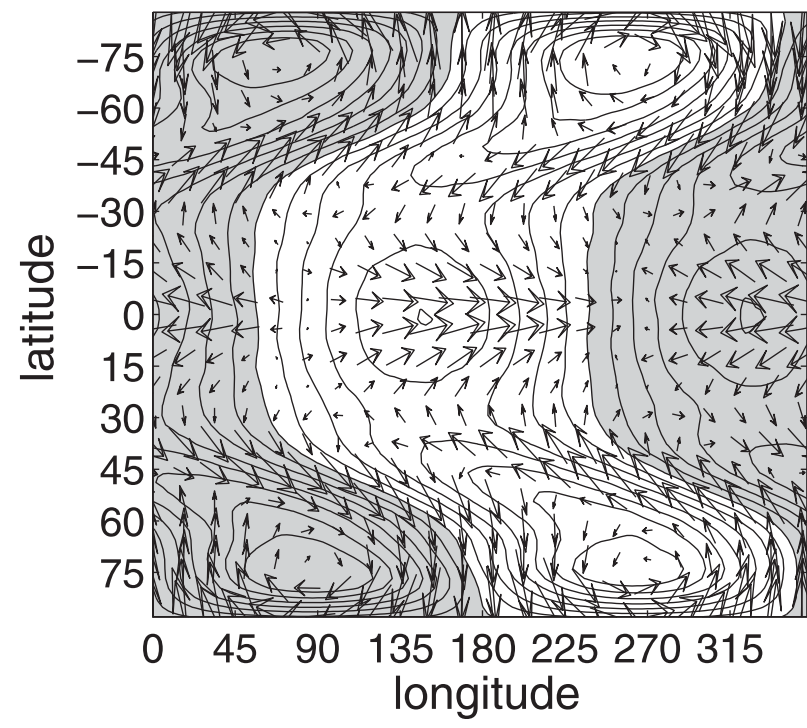

FIG. 16. Geopotential height (contours) and $u$ and $v$ (arrows) for the $R_{o}=1$ atmosphere at $475 \mathrm{hPa}$ regressed against an equatorial zonal wind time series filtered to retain only variability existing at wavenumber 1 with frequency between 0.17 and 0.37 day $^{-1}$. Contour level is $2 \mathrm{~m}$ with shaded regions negative. Maximum wind speed is $4.3 \mathrm{~m} \mathrm{~s}^{-1}$.

is due to the presence of a seasonal cycle (with westward momentum brought to the equator by the Hadley cell) and to a lesser extent the decelerating effects of Rossby wave breaking (Lee 1999). More generally, a strong seasonal cycle does not necessarily suppress superrotation if the overturning circulation is sufficiently weak, and the thermal damping time becomes another key parameter controlling superrotation (J. L. Mitchell et al. 2013, unpublished manuscript). Titan, for example, has both a strong seasonal cycle and strong superrotation.

We believe that the Kelvin wave mechanism discussed here provides a more robust explanation for dry dynamical core superrotation than the hypothesis of barotropic instability as discussed in MV10 and Williams (2003). As mentioned in the introduction, barotropic instability cannot bring eddy fluxes directly to the equator, since $\beta-u_{y y}$ cannot change sign there when the Northern and Southern Hemispheres are symmetric. The Kelvin wave mechanism allows for meridional eddy acceleration to be centered on the equator, a structure that can be seen in all of our superrotating runs (not shown).

One thing missing from our discussion is the source of the equatorial Kelvin waves that we see in the dry model. Figure 16 suggests that the equatorial Kelvin wave cannot be considered separately from the extratropical Rossby wave. Understanding of this coupled dynamic will hopefully be addressed in future studies. 
(a) zonal mean zonal wind
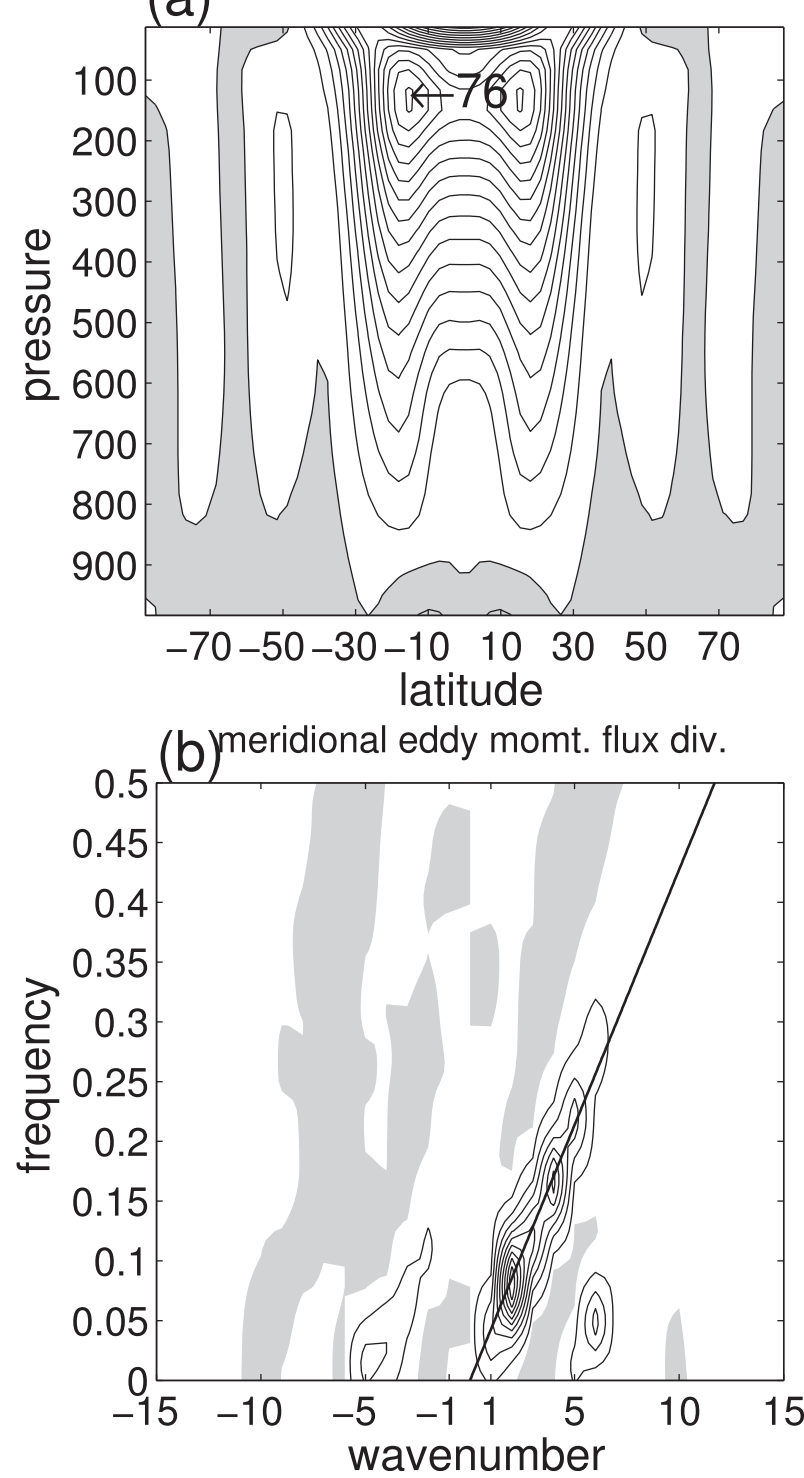

FIG. 17. For recreation of the $n=16$ atmosphere in Williams (2003). (a) Zonal-mean zonal wind (contour interval $5 \mathrm{~m} \mathrm{~s}^{-1}$ ). Shaded values are negative. Largest value is highlighted. (b) $\mathcal{S}$ in $\omega-k$ space at $550 \mathrm{hPa}$. Solid line shows the $H=40 \mathrm{~m}$ Kelvin wave dispersion relation. Shaded values are negative. Contour intervals are arbitrary.

Acknowledgments. The CLAUS figure results were obtained using the CLAUS archive held at the British Atmospheric Data Centre, produced using ISCCP source data distributed by the NASA Langley Data Center. NCEP Reanalysis II data provided by the NOAA/OAR/ESRL PSD, Boulder, Colorado, from their website at http://www.esrl.noaa.gov/psd. SFP and GKV were supported by the National Science Foundation under Grant AGS-1144302. JLM was supported by NASA Grant NNX12AI71G.

\section{REFERENCES}

Arnold, N. P., E. Tziperman, and B. Farrell, 2012: Abrupt transition to strong superrotation driven by equatorial wave resonance in an idealized GCM. J. Atmos. Sci., 69, 626-640.

Caballero, R., and M. Huber, 2010: Spontaneous transition to superrotation in warm climates simulated in CAM3. Geophys. Res. Lett., 37, L11701, doi:10.1029/2010GL043468.

Fels, S. B., and R. S. Lindzen, 1974: The interaction of thermally excited gravity waves with mean flows. Geophys. Fluid Dyn., 6, 149-191.

Hayashi, Y., 1982: Space-time spectral analysis and its application to atmospheric waves. J. Meteor. Soc. Japan, 60, 156-171.

Held, I. M., and A. H. Hou, 1980: Nonlinear axially symmetric circulations in a nearly inviscid atmosphere. J. Atmos. Sci., 37, 515-533.

— the dynamical cores of atmospheric general circulation models. Bull. Amer. Meteor. Soc., 75, 1825-1830.

Hendon, H. H., and M. C. Wheeler, 2008: Some space-time spectral analyses of tropical convection and planetary-scale waves. J. Atmos. Sci., 65, 2936-2948.

Heng, K., K. Menou, and P. J. Phillipps, 2011: Atmospheric circulation of tidally locked exoplanets: A suite of benchmark tests for dynamical solvers. Mon. Not. Roy. Astron. Soc., 413, 2380-2402.

Hide, R., 1969: Dynamics of the atmospheres of the major planets with an appendix on the viscous boundary layer at the rigid bounding surface of an electrically-conducting rotating fluid in the presence of a magnetic field. J. Atmos. Sci., 26, 841853.

Iga, S.-I., and Y. Matsuda, 2005: Shear instability in a shallow water model with implications for the Venus atmosphere. J. Atmos. Sci., 62, 2514-2527.

Imamura, T., 2006: The meridional propagation of planetary-scale waves in vertical shear: Implication for the Venus atmosphere. J. Atmos. Sci., 63, 1623-1636.

transport of zonal momentum due to Kelvin waves in a meridional circulation. J. Atmos. Sci., 61, 1966-1975.

Joshi, M., R. Haberle, and R. Reynolds, 1997: Simulations of the atmospheres of synchronously rotating terrestrial planets orbiting M dwarfs: Conditions for atmospheric collapse and the implications for habitability. Icarus, 129, 450-465.

Kasahara, A., 1980: Effect of zonal flows on the free oscillations of a barotropic atmosphere. J. Atmos. Sci., 37, 917-929.

Kiladis, G. N., M. C. Wheeler, P. T. Haertel, K. H. Straub, and P. E. Roundy, 2009: Convectively coupled equatorial waves. Rev. Geophys., 47, RG2003, doi:10.1029/2008RG000266.

Kraucunas, I., and D. L. Hartmann, 2005: Equatorial superrotation and the factors controlling the zonal-mean zonal winds in the tropical upper troposphere. J. Atmos. Sci., 62, 371-389.

Lee, S., 1999: Why are the climatological zonal winds easterly in the equatorial upper troposphere? J. Atmos. Sci., 56, 1353-1363.

Lian, Y., and A. P. Showman, 2010: Generation of equatorial jets by large-scale latent heating on the giant planets. Icarus, 207, 373-393.

Liu, J., and T. Schneider, 2011: Convective generation of equatorial superrotation in planetary atmospheres. J. Atmos. Sci., 68, 2742-2756.

Matsuno, T., 1966: Quasi-geostrophic motions in the equatorial area. J. Meteor. Soc. Japan, 44, 25-43. 
Merlis, T. M., and T. Schneider, 2010: Atmospheric dynamics of Earth-like tidally locked aquaplanets. J. Adv. Model. Earth Syst., 2 (13), doi:10.3894/JAMES.2010.2.13.

Mitchell, J. L., and G. K. Vallis, 2010: The transition to superrotation in terrestrial atmospheres. J. Geophys. Res., 115, E12008, doi:10.1029/2010JE003587.

Randel, W. J., and I. M. Held, 1991: Phase speed spectra of transient eddy fluxes and critical layer absorption. J. Atmos. Sci., 48, 688-697.

Rauscher, E., and K. Menou, 2012: A general circulation model for gaseous exoplanets with double-gray radiative transfer. $A s$ trophys. J., 750 (96), doi:10.1088/0004-637X/750/2/96.

Robinson, G. J., cited 2013: Cloud Archive User Service (CLAUS). NCAS British Atmospheric Data Centre. [Available online at http://badc.nerc.ac.uk/view/badc.nerc.ac.uk_ATOM_dataent_ claus.]

Saravanan, R., 1993: Equatorial superrotation and maintenance of the general circulation in two-level models. J. Atmos. Sci., 50, 1211-1227.

Schneider, T., 2006: The general circulation of the atmosphere. Annu. Rev. Earth Planet. Sci., 34, 655-688.

Showman, A. P., and L. M. Polvani, 2010: The Matsuno-Gill model and equatorial superrotation. Geophys. Res. Lett., 37, L18811, doi:10.1029/2010GL044343.

, and — 2011: Equatorial superrotation on tidally locked exoplanets. Astrophys. J., 738 (71), doi:10.1088/0004-637X/738/1/71.
J. J. Fortney, Y. Lian, M. S. Marley, R. S. Freedman, H. A. Knutson, and D. Charbonneau, 2009: Atmospheric circulation of hot Jupiters: Coupled radiative-dynamical general circulation model simulations of HD $189733 \mathrm{~b}$ and HD 209458b. Astrophys. J., 699 (564), doi:10.1088/0004-637X/ $699 / 1 / 564$.

Sobel, A. H., J. Nilsson, and L. M. Polvani, 2001: The weak temperature gradient approximation and balanced tropical moisture waves. J. Atmos. Sci., 58, 3650-3665.

Straub, K. H., and G. N. Kiladis, 2003: Extratropical forcing of convectively coupled Kelvin waves during austral winter. J. Atmos. Sci., 60, 526-543.

Suarez, M. J., and D. G. Duffy, 1992: Terrestrial superrotation: A bifurcation of the general circulation. J. Atmos. Sci., 49, $1541-1554$.

Vallis, G. K., 2006: Atmospheric and Oceanic Fluid Dynamics. Cambridge University Press, 745 pp.

Wheeler, M., and G. N. Kiladis, 1999: Convectively coupled equatorial waves: Analysis of clouds and temperature in the wavenumberfrequency domain. J. Atmos. Sci., 56, 374-399.

Williams, G., 2003: Barotropic instability and equatorial superrotation. J. Atmos. Sci., 60, 2136-2152.

Zhang, C., 2005: Madden-Julian oscillation. Rev. Geophys., 43, RG2003, doi:10.1029/2004RG000158.

Zhu, X., 2006: Maintenance of equatorial superrotation in the atmospheres of Venus and Titan. Planet. Space Sci., 54, 761-773. 